\title{
TURBULENCE-INDUCED RELATIVE VELOCITY OF DUST PARTICLES. II. THE BIDISPERSE CASE
}

\author{
Liubin Pan ${ }^{1}$, PaOlo Padoan ${ }^{2}$, and John Scalo $^{3}$ \\ ${ }^{1}$ Harvard-Smithsonian Center for Astrophysics, 60 Garden Street, Cambridge, MA 02138, USA; 1pan@ cfa.harvard.edu \\ ${ }^{2}$ ICREA and ICC, University of Barcelona, Marti i Franquès 1, E-08028 Barcelona, Spain; ppadoan@ icc.ub.edu \\ ${ }^{3}$ Department of Astronomy, University of Texas, Austin, TX 78712, USA; parrot@ astro.as.utexas.edu \\ Received 2014 February 25; accepted 2014 June 2; published 2014 July 25
}

\begin{abstract}
We extend our earlier work on turbulence-induced relative velocity between equal-size particles (Paper I, in this series) to particles of arbitrarily different sizes. The Pan \& Padoan (PP10) model shows that the relative velocity between different particles has two contributions, named the generalized shear and acceleration terms, respectively. The generalized shear term represents the particles' memory of the spatial flow velocity difference across the particle distance in the past, while the acceleration term is associated with the temporal flow velocity difference on individual particle trajectories. Using the simulation of Paper I, we compute the root-mean-square relative velocity, $\left\langle w^{2}\right\rangle^{1 / 2}$, as a function of the friction times, $\tau_{\mathrm{p} 1}$ and $\tau_{\mathrm{p} 2}$, of the two particles and show that the PP10 prediction is in satisfactory agreement with the data, confirming its physical picture. For a given $\tau_{\mathrm{p} 1}$ below the Lagrangian correlation time of the flow, $T_{\mathrm{L}},\left\langle w^{2}\right\rangle^{1 / 2}$ as a function of $\tau_{\mathrm{p} 2}$ shows a dip at $\tau_{\mathrm{p} 2} \simeq \tau_{\mathrm{p} 1}$, indicating tighter velocity correlation between similar particles. Defining a ratio $f \equiv \tau_{\mathrm{p}, 1} / \tau_{\mathrm{p}, \mathrm{h}}$, with $\tau_{\mathrm{p}, 1}$ and $\tau_{\mathrm{p}, \mathrm{h}}$ the friction times of the smaller and larger particles, we find that $\left\langle w^{2}\right\rangle^{1 / 2}$ increases with decreasing $f$ due to the generalized acceleration contribution, which dominates at $f \lesssim 1 / 4$. At a fixed $f$, our model predicts that $\left\langle w^{2}\right\rangle^{1 / 2}$ scales as $\tau_{\mathrm{p}, \mathrm{h}}^{1 / 2}$ for $\tau_{\mathrm{p}, \mathrm{h}}$ in the inertial range of the flow, stays roughly constant for $T_{\mathrm{L}} \lesssim \tau_{\mathrm{p}, \mathrm{h}} \lesssim T_{\mathrm{L}} / f$, and finally decreases as $\tau_{\mathrm{p}, \mathrm{h}}^{-1 / 2}$ for $\tau_{\mathrm{p}, \mathrm{h}} \gg T_{\mathrm{L}} / f$. The acceleration term is independent of the particle distance, $r$, and reduces the $r$ dependence of $\left\langle w^{2}\right\rangle^{1 / 2}$ in the bidisperse case.
\end{abstract}

Key words: minor planets, asteroids: general - planets and satellites: formation - protoplanetary disks - turbulence

Online-only material: color figures

\section{INTRODUCTION}

This paper is a follow-up to our earlier work on turbulenceinduced relative velocity of dust particles (Pan \& Padoan 2013; Paper I hereafter). The study is mainly motivated by the problem of dust particle growth and planetesimal formation in protoplanetary disks (e.g., Dullemond \& Dominik 2005; Blum \& Wurm 2008; Guttler et al. 2010; Zsom et al. 2010, 2011; Birnstiel et al. 2011; Windmark et al. 2012; Garaud et al. 2013). In Paper I, we conducted an extensive statistical study of the relative velocity and the collision kernel of equal-size particles suspended in turbulent flows using both analytical and numerical methods. The case of equal-size particles, usually referred to as the monodisperse case, is of theoretical interest, but insufficient for astrophysical applications, as dust particles in protoplanetary disks have a size distribution. The main goal of the current paper is to investigate turbulence-induced relative velocity in the general case of different particles of arbitrary sizes, known as the bidisperse case.

Saffman \& Turner (1956, hereafter S-T) derived a formula for the variance of the turbulence-induced relative velocity in the limit of small particles with friction time, $\tau_{\mathrm{p}}$, much smaller than the Kolmogorov timescale, $\tau_{\eta}$. This limit, known as the S-T limit, is usually expressed as $\mathrm{St} \ll 1$, where the Stokes number, St, is defined as $\mathrm{St} \equiv \tau_{\mathrm{p}} / \tau_{\eta}$. The $\mathrm{S}$-T formula consists of two terms, named the shear and the acceleration term, respectively (e.g., Zhou et al. 2001). The shear term is determined solely by the flow velocity difference across the particle distance, $r$. It is independent of St but has a significant dependence on $r$ (see Paper I). The name of the acceleration term originates from its dependence on the acceleration, $\boldsymbol{a}$, of the flow velocity, and it contributes a one-dimensional (1D) variance of $a^{2}\left(\tau_{\mathrm{p} 2}-\tau_{\mathrm{p} 1}\right)^{2}$ to the relative velocity, where $\tau_{\mathrm{p} 1}$ and $\tau_{\mathrm{p} 2}$ are the friction times of the two particles, and $a$ is the $1 \mathrm{D} \mathrm{rms}$ of the flow acceleration. This effect of the flow acceleration on the relative velocity of small particles of different sizes was also found by Weidenschilling (1984). In the monodisperse case, the acceleration term vanishes, and only the shear term contributes. The shear term in the S-T prediction for equal-size particles has been discussed in detail in Paper I. Its validity, accuracy, and limitations have been systematically examined using a numerical simulation.

In the bidisperse case, the fundamental difference from the case of equal-size particles is the contribution of the acceleration term, which tends to increase the particle collision velocity. The dependence of the acceleration term on the friction time difference, $\tau_{\mathrm{p} 2}-\tau_{\mathrm{p} 1}$, corresponds to the fact that particles of different sizes have different responses to the flow velocities along their trajectories. Interestingly, unlike the shear term, the acceleration contribution in the S-T formula is independent of the particle distance, $r$. This observation is of particular importance for the application to dust particles in protoplanetary disks. Because the size of dust particles is typically much smaller than the Kolmogorov scale, $\simeq 1 \mathrm{~km}$, of protoplanetary turbulence, one is required to examine the collisional statistics in the $r \rightarrow 0$ limit (e.g., Hubbard 2012, 2013) that are not accessible to numerical simulations due to their limited resolution. Therefore, unless the measured statistics already converge at the resolution scale, an extrapolation to the $r \rightarrow$ 0 limit is needed. Such an extrapolation was found to be challenging for small equal-size particles with $\mathrm{St} \lesssim 1$ due to the $r$ dependence of the shear effect ${ }^{4}$ (Paper I). In the bidisperse case, the presence of the acceleration term reduces the $r$ dependence

\footnotetext{
4 To evaluate the collision kernel of small equal-size particles in the $r \rightarrow 0$ limit, a method is developed in Paper I to isolate an $r$-independent contribution by splitting particle pairs at given distances into two types, named continuous
} 
and makes it easier to achieve numerical convergence for the relative velocity between small particles of different sizes.

Pan \& Padoan (2010; PP10) developed a model for the rms relative velocity in the general bidisperse case for arbitrarily different particles of any size. It was shown that the model prediction is in good agreement with the simulation data of Zhou et al. (2001) at low resolutions. The PP10 formulation for the relative velocity also consists of two contributions, named the generalized shear and acceleration terms, as they reduce to the shear and acceleration terms, respectively, in the S-T formula in the small-particle limit. It can thus be viewed as a generalized formulation that extends the $\mathrm{S}-\mathrm{T}$ limit $\left(\mathrm{St}_{1,2} \ll 1\right)$ to particles of arbitrary size. The generalized shear term has a form similar to the monodisperse model discussed in Paper I. It represents the particles' memory of the spatial flow velocity difference, $\Delta \boldsymbol{u}(R)$, across the separation, $R$, of the two particles at given times in the past. The physical meaning of the generalized acceleration term will be clarified in the present paper, and we will show its connection with the temporal flow velocity difference, $\Delta_{\mathrm{T}} \boldsymbol{u}$, along individual trajectories of the two particles. An approximate relation for the acceleration term will be established in terms of $\Delta_{\mathrm{T}} \boldsymbol{u}$ and the particle friction times. Using the simulation of Paper I, we will systematically test the PP10 model for the relative velocity between different particles.

A variety of models have been developed to predict the relative velocity of particles of different sizes, covering the entire scale range of the turbulent flow (e.g., Völk et al. 1980; Yuu 1984; Kruis \& Kusters 1997; Zhou et al. 2001; Zaichik \& Alipchenkov 2003, 2009; Zaichik et al. 2003, 2006, 2008; see PP10 and references therein). In the astrophysics literature, the model of choice has been that by Völk et al. (1980) and its later refinements (e.g., Markiewicz et al. 1991; Cuzzi \& Hogan 2003; Ormel \& Cuzzi 2007). In this paper, we only test the PP10 model in an effort to provide an improved physical insight. Other models, particularly Völk et al. (1980) and Zaichik et al. (2008), will be tested and compared with PP10 in a separate work.

Theoretical models only predict the rms or variance of the relative velocity, which, however, is not sufficient to model collisions of dust particles (Paper I). In fact, the rms does not directly enter the estimate of the collision kernel, which is determined by the first-order moments, i.e., the average of the radial component or the mean three-dimensional (3D) amplitude of the relative velocity (Wang et al. 2000). The variance of the relative velocity does not even represent the average collision energy per collision. Instead, using a collision-rate weighting, the average collision energy depends on the third-order moment of the collision velocity (e.g., Hubbard 2012). Furthermore, an accurate coagulation model for dust particles in protoplanetary disks requires the entire probability distribution of the collision velocity, as the outcome of each collision depends on the collision velocity (Windmark et al. 2012; Garaud et al. 2013). Despite these limitations, the rms relative velocity still provides a rough approximation to the mean of the relative velocity, and it is therefore a useful tool to shed light on the physics of turbulence-induced particle collisions. The main purpose of the current work is to confirm the accuracy of the PP10 model for the rms relative velocity and hence to validate the physical picture revealed by that model. We will show in a separate paper that this physical picture provides an understanding of the probability distribution of the collision velocity as well.

(S-T) and caustic (sling) pairs, respectively (Falkovich et al. 2002; Wilkinson et al. 2006).
In addition to turbulent motions, there are other effects, such as differential settling or radial drift, that can provide important contributions to the relative velocity between dust particles of different sizes in protoplanetary disks. In this work, we do not consider these contributions. The numerical experiment used here employs a statistically stationary and isotropic turbulent flow, which is a further idealization relative to realistic protoplanetary disks with Keplerian rotation, stratifications, etc. However, the highly idealized simulation provides a useful tool to study the role of turbulence-induced collisions.

In Section 2, we present a simple model for the relative velocity between inertial particles and the local flow velocity, a special bidisperse case that provides a clean comparison between our model and the simulation. The PP10 formulation for the bidisperse relative velocity is reviewed in Section 3. A brief presentation of our numerical simulation is given in Section 4. In Section 5, we examine the statistics of the particleflow relative velocity. In Section 6, we show simulation results for the rms relative velocity and test the prediction of the PP10 model. We summarize the main results and conclusions in Section 7.

\section{THE PARTICLE-FLOW RELATIVE VELOCITY}

We first consider a simple model for the relative velocity between an inertial particle and the local flow element. This is a special bidisperse case where one of the particles is a tracer with zero inertia. It provides a useful illustration for the general bidisperse case. The particle-flow relative velocity is also of interest for practical application, e.g., in the formation of fine dust rims of chondrules via an accretion process (e.g., Paque \& Cuzzi 1997; Morfill et al. 1998; Cuzzi \& Hogan 2003; Ormel et al. 2008; Carballido 2011). The velocity $\boldsymbol{v}(t)$ of an inertial particle with a friction timescale $\tau_{\mathrm{p}}$ evolving in a turbulent velocity field $\boldsymbol{u}(\boldsymbol{x}, t)$ is governed by the equation

$$
\frac{d \boldsymbol{v}}{d t}=\frac{\boldsymbol{u}(\boldsymbol{X}(t), t)-\boldsymbol{v}}{\tau_{\mathrm{p}}},
$$

where $\boldsymbol{X}(t)$ is the particle position and $\boldsymbol{u}(\boldsymbol{X}(t), t)$ is the flow velocity "seen" by the particle. Equation (1) is a stochastic differential equation in a form similar to the Langevin equation, with the flow velocity acting as a random force. However, it differs from the Langevin equation in that the correlation time of the "force" is significant in comparison with the friction time, $\tau_{\mathrm{p}}$. Equation (1) can be formally integrated, as if it were a deterministic equation. As shown in Paper I, the particle velocity at any given time, e.g., $t=0$, can be evaluated as

$$
\boldsymbol{v}(0)=\frac{1}{\tau_{\mathrm{p}}} \int_{t_{0}}^{0} \boldsymbol{u}(\boldsymbol{X}(\tau), \tau) \exp \left(\frac{\tau}{\tau_{\mathrm{p}}}\right) d \tau,
$$

where it is assumed that at $t=0$ the particle has already forgotten its inertial velocity at $t_{0}$. This is equivalent to assuming $t_{0} \ll-\tau_{\mathrm{p}}$, which also allows us to replace the lower limit, $t_{0}$, by $-\infty$ (Paper I).

At time $t=0$, we define a flow-particle relative velocity at $\boldsymbol{x}$ as $\boldsymbol{w}_{\mathrm{f}}(\boldsymbol{x}, 0) \equiv \boldsymbol{u}(\boldsymbol{x}, 0)-\boldsymbol{v}(0)$. Using the formal solution, we have

$$
\boldsymbol{w}_{\mathrm{f}}=\frac{1}{\tau_{\mathrm{p}}} \int_{-\infty}^{0}[\boldsymbol{u}(\boldsymbol{x}, 0)-\boldsymbol{u}(\boldsymbol{X}(\tau), \tau)] \exp \left(\frac{\tau}{\tau_{\mathrm{p}}}\right) d \tau
$$

where $\boldsymbol{X}(\tau)$ satisfies the condition $\boldsymbol{X}(0)=\boldsymbol{x}$. The equation suggests that the particle-flow relative velocity depends on the 
difference between the local flow velocity and the velocity the particle saw within a friction timescale in the past. If we define a flow velocity difference, $\Delta_{\mathrm{T}} \boldsymbol{u}(\Delta \tau)$, at a time lag, $\Delta \tau$, along the particle trajectory, $\boldsymbol{w}_{\mathrm{f}}$ can be roughly estimated as $\boldsymbol{w}_{\mathrm{f}} \simeq \Delta_{\mathrm{T}} \boldsymbol{u}\left(\tau_{\mathrm{p}}\right)$.

The variance of $\boldsymbol{w}_{\mathrm{f}}$ can be calculated as

$$
\begin{aligned}
\left\langle w_{\mathrm{fi} i} w_{\mathrm{f} j}\right\rangle= & \int_{-\infty}^{0} \frac{d \tau}{\tau_{\mathrm{p}}} \int_{-\infty}^{0} \frac{d \tau^{\prime}}{\tau_{\mathrm{p}}} \\
& \times\left[B_{i j}(0,0)-B_{i j}\left(0, \tau^{\prime}\right)-B_{i j}(\tau, 0)+B_{i j}\left(\tau, \tau^{\prime}\right)\right] \\
& \times \exp \left(\frac{\tau}{\tau_{\mathrm{p}}}\right) \exp \left(\frac{\tau^{\prime}}{\tau_{\mathrm{p}}}\right)
\end{aligned}
$$

where the trajectory correlation tensor, $B_{i j}\left(\tau, \tau^{\prime}\right) \equiv$ $\left\langle u_{i}(\boldsymbol{X}(\tau), \tau) u_{j}\left(\boldsymbol{X}\left(\tau^{\prime}\right), \tau^{\prime}\right)\right\rangle$, corresponds to a two-time correlation of the flow velocity along the particle trajectory (Paper I). The integral of the fourth term, $B_{i j}\left(\tau, \tau^{\prime}\right)$, can be simplified using the fact that $B_{i j}\left(\tau, \tau^{\prime}\right)$ is an even function of the time lag, $\Delta \tau=\tau^{\prime}-\tau$, i.e., $B_{i j}\left(\tau, \tau^{\prime}\right)=B_{i j}(|\Delta \tau|)$, in statistically stationary turbulence. Making a variable change, $\xi=\tau+\tau^{\prime}$ and $\zeta=\Delta \tau=\tau^{\prime}-\tau$, for this term, we find

$$
\begin{aligned}
\left\langle w_{\mathrm{f} i} w_{\mathrm{f} j}\right\rangle & =\frac{1}{\tau_{\mathrm{p}}} \int_{-\infty}^{0}\left[B_{i j}(0)-B_{i j}(\Delta \tau)\right] \exp \left(\frac{\Delta \tau}{\tau_{\mathrm{p}}}\right) d \Delta \tau \\
& =\frac{1}{2 \tau_{\mathrm{p}}} \int_{-\infty}^{0} D_{i j}(\Delta \tau) \exp \left(\frac{\Delta \tau}{\tau_{\mathrm{p}}}\right) d \Delta \tau
\end{aligned}
$$

where $D_{i j}(\Delta \tau) \equiv\left\langle\Delta_{\mathrm{T}} u_{i}(\Delta \tau) \Delta_{\mathrm{T}} u_{j}(\Delta \tau)\right\rangle$ is the temporal structure function of the flow velocity along the particle trajectory.

For isotropic turbulence, $B_{i j}(\Delta \tau)=u^{\prime 2} \Phi_{1}(\Delta \tau) \delta_{i j}$, where $u^{\prime}$ is the $1 \mathrm{D}$ rms velocity of the turbulent flow, and $\Phi_{1}$ is the trajectory correlation function. It follows that $D_{i j}(\Delta \tau)=$ $2 u^{\prime 2} \delta_{i j}\left(1-\Phi_{1}(\Delta \tau)\right)$. A common assumption for $\Phi_{1}$ is to approximate it by the Lagrangian temporal correlation function $\Phi_{\mathrm{L}}$ (see, e.g., Zaichik et al. 2006, 2008; Derivich 2006). This is equivalent to approximating $D_{i j}(\Delta \tau)$ in Equation (5) by the Lagrangian structure tensor, $D_{\mathrm{L} i j}(\Delta \tau)=D_{\mathrm{L}}(\Delta \tau) \delta_{i j}$, with $D_{\mathrm{L}}=2 u^{\prime 2}\left(1-\Phi_{\mathrm{L}}(\Delta \tau)\right)$. For very large particles, it may be a better assumption to set $D_{i j}(\Delta \tau)$ to the Eulerian temporal structure tensor $D_{\mathrm{E} i j}(\Delta \tau)$ (see the definition in Appendix C).

In Paper I, we considered single- and bi-exponential forms for $\Phi_{\mathrm{L}}$. The exponential form is $\Phi_{\mathrm{L}}(\Delta \tau)=\exp \left(-|\Delta \tau| / T_{\mathrm{L}}\right)$, where $T_{\mathrm{L}}$ is the Lagrangian correlation timescale. Setting $\Phi_{1}(\Delta \tau)=\exp \left(-|\Delta \tau| / T_{\mathrm{L}}\right)$ and integrating Equation (5), we obtain $\left\langle w_{\mathrm{f} i} w_{\mathrm{f} j}\right\rangle=\left(w_{\mathrm{f}}^{\prime}\right)^{2} \delta_{i j}$, where the $1 \mathrm{D} \mathrm{rms}, w_{\mathrm{f}}^{\prime}$, of the flowparticle relative velocity is given by

$$
w_{\mathrm{f}}^{\prime}=u^{\prime}\left(\frac{\tau_{\mathrm{p}}}{\tau_{\mathrm{p}}+T_{\mathrm{L}}}\right)^{1 / 2},
$$

which indicates a $\tau_{\mathrm{p}}^{1 / 2}$ scaling for particles with $\tau_{\mathrm{p}} \ll T_{\mathrm{L}}$. In the large-particle limit $\left(\tau_{\mathrm{p}} \gg T_{\mathrm{L}}\right), w_{\mathrm{f}}^{\prime}$ approaches $u^{\prime}$ as the particle velocity becomes much smaller than the flow velocity.

As discussed in Paper $\mathrm{I}, \Phi_{\mathrm{L}}(\Delta \tau)$ is better fit by a biexponential form (see Figure 2 of Paper I),

$$
\begin{aligned}
\Phi_{\mathrm{L}}(\Delta \tau)= & \frac{1}{2 \sqrt{1-2 z^{2}}}\left\{\left(1+\sqrt{1-2 z^{2}}\right) \exp \left[-\frac{2|\Delta \tau|}{\left(1+\sqrt{1-2 z^{2}}\right) T_{\mathrm{L}}}\right]\right. \\
& \left.-\left(1-\sqrt{1-2 z^{2}}\right) \exp \left[-\frac{2|\Delta \tau|}{\left(1-\sqrt{1-2 z^{2}}\right) T_{\mathrm{L}}}\right]\right\}
\end{aligned}
$$

where the parameter $z\left(\equiv \tau_{\mathrm{T}} / T_{\mathrm{L}}\right)$ is the ratio of the Taylor microtimescale, $\tau_{\mathrm{T}}$, to $T_{\mathrm{L}}$. The Taylor (Lagrangian) timescale is defined as $\tau_{\mathrm{T}}=\left(2 u^{\prime 2} / a^{2}\right)^{1 / 2}$ with $a$ the $1 \mathrm{D} \mathrm{rms}$ of the acceleration field, $\boldsymbol{a}$, of the flow. The theoretical motivation of adopting a bi-exponential function is that, by accounting for the flow acceleration, it correctly reflects the smoothness of $\Phi_{\mathrm{L}}(\Delta \tau)$ at small $\Delta \tau\left(\lesssim \tau_{\mathrm{T}}\right)$. For $\Delta \tau \ll \tau_{\mathrm{T}}$, the bi-exponential function ensures that the temporal velocity difference along a Lagrangian trajectory scales linearly with $\Delta \tau$ and thus correctly describes the dissipation range in the Lagrangian frame (Zaichik et al. 2006). In other words, the bi-exponential function reflects the transition from the dissipation-range scaling to the inertial-range scaling in the Lagrangian frame. On the other hand, the single exponential ignores the existence of the dissipation range and is thus physically inadequate. The parameter $z$ in Equation (7) is essentially a measure of the width of the inertial range in the Lagrangian coordinate. Approximately, $z^{2} \sim \tau_{\eta} / T_{\mathrm{L}}$ with $\tau_{\eta}$ the Kolmogorov time, and it is thus an indicator of the timescale separation between the dissipation and the driving scales. Clearly, $z$ decreases with the flow Reynolds number $(\mathrm{Re})$, and it roughly scales with $\operatorname{Re}$ as $z \propto \operatorname{Re}^{-1 / 4}$. With Equation (7) for $\Phi_{\mathrm{L}}$, we find

$$
w_{\mathrm{f}}^{\prime}=u^{\prime}\left(\frac{\Omega^{2}}{\Omega+\Omega^{2}+z^{2} / 2}\right)^{1 / 2},
$$

where $\Omega \equiv \tau_{\mathrm{p}} / T_{\mathrm{L}}$. Equation (8) shows that $w_{\mathrm{f}}^{\prime}$ approaches $u^{\prime}$ for $\Omega \gg 1$ and scales with $\tau_{\mathrm{p}}$ as $\tau_{\mathrm{p}}^{1 / 2}$ for intermediate particles with $z^{2} / 2 \ll \Omega \ll 1$. For small particles with $\Omega \ll z^{2} / 2$ (i.e., $\tau_{\mathrm{p}} \ll \tau_{\mathrm{T}}^{2} / 2 T_{\mathrm{L}} \simeq \tau_{\eta}$ ), Equation (8) predicts $w_{\mathrm{f}}^{\prime}=\sqrt{2} u^{\prime} \Omega / z \equiv a \tau_{\mathrm{p}}$ (see also Weidenschilling 1984). This is expected from Equation (1), assuming $d \boldsymbol{v} / d t \simeq d \boldsymbol{u} / d t=\boldsymbol{a}$. The linear scaling of $w_{\mathrm{f}}^{\prime}$ with $\tau_{\mathrm{p}}$ for $\mathrm{St} \ll 1$ particles cannot be correctly captured by Equation (6), which uses a singleexponential form for $\Phi_{1}$ or $\Phi_{\mathrm{L}}$. We will compare the prediction, Equation (8), against our simulation data in Section 5.

\section{THE PARTICLE RELATIVE VELOCITY IN THE BIDISPERSE CASE}

Following PP10 and Paper I, we use superscripts (1) and (2) to label two particles coming together. In the bidisperse case, we denote the friction times of the two particles as $\tau_{\mathrm{p} 1}$ and $\tau_{\mathrm{p} 2}$. At a given time $t$, we examine the relative velocity, $\boldsymbol{w} \equiv \boldsymbol{v}^{(1)}(t)-\boldsymbol{v}^{(2)}(t)$, of particle pairs at given separations, $\boldsymbol{r}$. The particle positions at $t$ are constrained by $\boldsymbol{X}^{(1)}(t)-\boldsymbol{X}^{(2)}(t)=\boldsymbol{r}$. We denote the flow velocities seen by the two particles as $\boldsymbol{u}^{(1)}(t)$ $\left(\equiv \boldsymbol{u}\left(\boldsymbol{X}^{(1)}, t\right)\right.$ ) and $\boldsymbol{u}^{(2)}(t)$. We characterize the second-order statistics of $\boldsymbol{w}$ by a particle velocity structure tensor,

$$
S_{\mathrm{p} i j}(\boldsymbol{r})=\left\langle w_{i} w_{j}\right\rangle=\left\langle\left(v_{i}^{(1)}-v_{i}^{(2)}\right)\left(v_{j}^{(1)}-v_{j}^{(2)}\right)\right\rangle,
$$

where $\langle\cdots\rangle$ denotes the ensemble average. From statistical homogeneity and stationarity, $S_{\mathrm{p} i j}$ depends only on $\boldsymbol{r}$. Further assuming isotropy, it can be written as (Paper I)

$$
S_{\mathrm{p} i j}(\boldsymbol{r})=\left\langle w_{\mathrm{t}}^{2}\right\rangle \delta_{i j}+\left(\left\langle w_{\mathrm{r}}^{2}\right\rangle-\left\langle w_{\mathrm{t}}^{2}\right\rangle\right) \frac{r_{i} r_{j}}{r^{2}},
$$

where $\left\langle w_{\mathrm{r}}^{2}\right\rangle$ and $\left\langle w_{\mathrm{t}}^{2}\right\rangle$ are the variances of the radial component, $w_{r}$ ( $\left.\equiv w_{i} r_{i} / r\right)$, and a tangential component, $w_{t}$, of the relative velocity, respectively. From Equation (10), we see that $\left\langle w_{\mathrm{r}}^{2}\right\rangle=$ $S_{\mathrm{p} i j} r_{i} r_{j} / r^{2}$, and the $3 \mathrm{D}$ variance, $\left\langle w^{2}\right\rangle=S_{\mathrm{p} i i}=\left\langle w_{\mathrm{r}}^{2}\right\rangle+2\left\langle w_{\mathrm{t}}^{2}\right\rangle$. 


\subsection{The Limits of Small and Large Particles}

Saffman \& Turner (1956) studied turbulence-induced relative velocity in the small-particle limit with $\tau_{\mathrm{p} 1}, \tau_{\mathrm{p} 2}$ much smaller than the Kolmogorov timescale, $\tau_{\eta}$, of the turbulent flow or with Stokes numbers $\mathrm{St}_{1,2} \equiv \tau_{\mathrm{p} 1,2} / \tau_{\eta} \ll 1$. Using a Taylor expansion of Equation (1) for these small particles to calculate $S_{\text {pij }}$ gives (Saffman \& Turner 1956)

$$
S_{\mathrm{p} i j}(\boldsymbol{r})=\left\langle a_{i} a_{j}\right\rangle\left(\tau_{\mathrm{p} 1}-\tau_{\mathrm{p} 2}\right)^{2}+S_{i j}(\boldsymbol{r}),
$$

where $\boldsymbol{a}$ is the local flow acceleration, and the flow structure tensor, $S_{i j}(\boldsymbol{r})$, is defined as $S_{i j}(\boldsymbol{r}) \equiv\left\langle\Delta u_{i} \Delta u_{j}\right\rangle$ with $\Delta u_{i}$ the velocity difference, $u_{i}(\boldsymbol{x}+\boldsymbol{r})-u_{i}(\boldsymbol{x})$, across $\boldsymbol{r}$. In an isotropic flow, $S_{i j}=S_{\mathrm{nn}} \delta_{i j}+\left(S_{\mathrm{ll}}-S_{\mathrm{nn}}\right) r_{i} r_{j} / r^{2}$, where $S_{\mathrm{ll}}(r)$ and $S_{\mathrm{nn}}(r)$ are the longitudinal and transverse structure functions (Paper I). For $r$ below the Kolmogorov scale, $\eta\left(\equiv\left(v^{3} \bar{\epsilon}\right)^{1 / 4}\right)$, of an incompressible flow, $S_{\mathrm{ll}}(r)=(1 / 15)(\bar{\epsilon} / \nu) r^{2}$ and $S_{\mathrm{nn}}(r)=$ $(2 / 15)(\bar{\epsilon} / v) r^{2}$, respectively (Monin \& Yaglom 1975). Here $v$ and $\bar{\epsilon}$ are the viscosity and the average energy dissipation rate of the flow.

With $\left\langle a_{i} a_{j}\right\rangle=a^{2} \delta_{i j}$ from isotropy, we find by comparing Equation (11) with Equation (10) that

$$
\begin{aligned}
& \left\langle w_{\mathrm{r}}^{2}\right\rangle=\left(\tau_{\mathrm{p} 1}-\tau_{\mathrm{p} 2}\right)^{2} a^{2}+\frac{1}{15} \frac{\bar{\epsilon}}{\nu} r^{2}, \\
& \left\langle w_{\mathrm{t}}^{2}\right\rangle=\left(\tau_{\mathrm{p} 1}-\tau_{\mathrm{p} 2}\right)^{2} a^{2}+\frac{2}{15} \frac{\bar{\epsilon}}{v} r^{2},
\end{aligned}
$$

where the first and second terms on the right-hand sides are usually referred to as the acceleration and shear terms, respectively (Wang et al. 2000; Zhou et al. 2001). In the monodisperse case, the acceleration terms vanish, and only the shear terms contribute. The accuracy and the weakness of the shear terms in the S-T formula for equal-size particles have been discussed in Paper I. ${ }^{5}$ The acceleration terms depend on the friction time difference (see also Weidenschilling 1984), and unlike the shear terms, they are independent of $r$.

We next consider the opposite limit of large particles with $\tau_{\mathrm{p} 1,2} \gg T_{\mathrm{L}}$, with $T_{\mathrm{L}}$ the Lagrangian correlation time of the flow. Motions of these particles are similar to Brownian motion, and the velocities of any such particles are uncorrelated. Therefore, $S_{\mathrm{p} i j}(\boldsymbol{r})=\left[\left(v^{\prime(1)}\right)^{2}+\left(v^{\prime(2)}\right)^{2}\right] \delta_{i j}$, where $v^{\prime(1)}$ and $v^{\prime(2)}$ are the oneparticle rms velocities (see Paper I). In this limit, we have

$$
\left\langle w_{\mathrm{r}}^{2}\right\rangle=\left\langle w_{\mathrm{t}}^{2}\right\rangle=u^{\prime 2}\left(\frac{1}{\Omega_{1}}+\frac{1}{\Omega_{2}}\right),
$$

where $\Omega_{1,2}=\tau_{\mathrm{p} 1,2} / T_{\mathrm{L}}$, and we used $v^{\prime(1,2)}=u^{\prime} \Omega_{1,2}^{-1 / 2}$ for $\Omega_{1,2} \gg 1$ (Abrahamson 1975).

An interesting limiting case is when only one of the particles is very large, with $\tau_{\mathrm{p}} \gg T_{\mathrm{L}}$. The velocity of this particle is small $\left(\ll u^{\prime}\right)$, and its relative velocity with the small particle would be approximately the one-particle velocity of the small particle. The one-particle velocity has been examined in Paper I. If the small particle has $\tau_{\mathrm{p}} \ll T_{\mathrm{L}}$, its velocity is close to the flow rms velocity, $u^{\prime}$, and the relative speed with the large particle is expected to be $\simeq u^{\prime}$. The flow-particle relative velocity discussed in Section 2 is a special limiting case with one of the particles being a tracer $(\mathrm{St}=0)$.

\footnotetext{
5 For example, the S-T formula predicts that the shear term for $\left\langle w_{\mathrm{t}}^{2}\right\rangle$ is two times larger than that for $\left\langle w_{\mathrm{r}}^{2}\right\rangle$. But our simulation shows that the radial and tangential rms relative speeds are nearly equal for equal-size particles with $\mathrm{St} \gtrsim 0.1$. We also found that the linear scaling of the rms relative speed with $r$ predicted by $\mathrm{S}$-T for equal-size particles does not hold for $\mathrm{St} \gtrsim 0.1$, due to the sling effect or caustic formation (Paper I). The S-T formula for shear terms may have higher accuracy at $\mathrm{St} \ll 0.1$.
}

\subsection{The Formulation of Pan \& Padoan (2010)}

We briefly summarize the formulation of the model by Pan $\&$ Padoan (2010; hereafter PP10) for the relative velocity variance in the bidisperse case. The monodisperse version of the model for equal-size particles was presented in Paper I. The model is derived by calculating $S_{\mathrm{p} i j}(\boldsymbol{r})$ in Equation (9) using the formal solution, Equation (2), for the particle velocity. The derivation is reviewed in Appendix A, where an assumption implicitly made in PP10 is pointed out and justified. In the general PP10 model, $S_{\mathrm{p} i j}$ can be written as two terms,

$$
S_{\mathrm{p} i j}(\boldsymbol{r})=\mathcal{A}_{i j}+\mathcal{S}_{i j}
$$

which reduce to the acceleration and shear terms, respectively, in the S-T limit, Equation (12) (see Appendix A and Section 3.2.1). The formulation may thus be viewed as a generalization of the $\mathrm{S}$-T formula for particles of any arbitrary size. We name $\mathcal{A}_{i j}$ and $\mathcal{S}_{i j}$ the generalized acceleration and shear terms, respectively (PP10). Equations for the two terms are given in Appendix A (Equations (A6) and (A7)). Note that the generalized shear term was denoted as $\mathcal{D}_{i j}$ in PP10.

Like the acceleration term in the $\mathrm{S}$-T formula, $\mathcal{A}_{i j}$ vanishes for particles of equal size. $\mathcal{A}_{i j}$ relies only on the flow velocity statistics along individual trajectories of the two particles and is thus independent of $\boldsymbol{r}$. The generalized shear term represents the contribution from the particles' memory of the flow velocity difference they saw in the past, and it can be modeled in a way similar to the case of equal-size particles discussed in Paper I. Due to the complexity of the problem, our formulation is complicated (see Appendix A) and not straightforward for applications. In further works, we will establish simple function fits or simplified forms that agree with our data and are practically easy to use. In this paper, we focus on testing the accuracy of the model.

It is interesting to split the relative velocity, $\boldsymbol{w}$, into an acceleration component, $\boldsymbol{w}_{\mathrm{a}}$, and a shear component, $\boldsymbol{w}_{\mathrm{s}}$, such that $\left\langle w_{\mathrm{a} i} w_{\mathrm{a} j}\right\rangle=\mathcal{A}_{i j}$ and $\left\langle w_{\mathrm{s} i} w_{\mathrm{s} j}\right\rangle=\mathcal{S}_{i j}$. It is implicitly assumed that $\boldsymbol{w}_{\mathrm{a}}$ and $\boldsymbol{w}_{\mathrm{s}}$ are statistically independent. We discuss the modeling and physical meaning of the generalized acceleration and shear effects in the next two subsections.

\subsubsection{The Generalized Acceleration Term}

To understand the physical meaning of the generalized acceleration term, we use an approximation for the Equation (A6) of $\mathcal{A}_{i j}$ derived in Appendix A. The term depends on the temporal flow velocity correlations, $\left\langle u_{i}^{(1)}(\tau) u_{j}^{(1)}\left(\tau^{\prime}\right)\right\rangle$ and $\left\langle u_{i}^{(2)}(\tau) u_{j}^{(2)}\left(\tau^{\prime}\right)\right\rangle$, along the individual trajectories of the two particles. Assuming that the trajectory statistics of the two particles are equivalent, i.e., $\left\langle u_{i}^{(1)}(\tau) u_{j}^{(1)}\left(\tau^{\prime}\right)\right\rangle \simeq\left\langle u_{i}^{(2)}(\tau) u_{j}^{(2)}\left(\tau^{\prime}\right)\right\rangle$, Equation (A6) can be rewritten as

$$
\begin{aligned}
\mathcal{A}_{i j} \simeq & \int_{-\infty}^{0} \frac{d \tau}{\tau_{\mathrm{p} 2}} \int_{-\infty}^{0} \frac{d \tau^{\prime}}{\tau_{\mathrm{p} 2}}\left\langle\left[u_{i}^{(2)}(\tau)-u_{i}^{(2)}(f \tau)\right]\right. \\
& \left.\times\left[u_{j}^{(2)}\left(\tau^{\prime}\right)-u_{j}^{(2)}\left(f \tau^{\prime}\right)\right]\right\rangle \exp \left(\frac{\tau}{\tau_{\mathrm{p} 2}}\right) \exp \left(\frac{\tau^{\prime}}{\tau_{\mathrm{p} 2}}\right),
\end{aligned}
$$

where $f \equiv \tau_{\mathrm{p} 1} / \tau_{\mathrm{p} 2}$ is the friction time ratio of the two particles. Without loss of generality, we assume $\tau_{\mathrm{p} 1} \leqslant \tau_{\mathrm{p} 2}$ or $f \leqslant 1$. Throughout the paper, we define $f$ as the friction time (or Stokes number) ratio of the smaller particle to the larger one. Note that Equation (15) depends on the flow velocity, $\boldsymbol{u}^{(2)}$, 


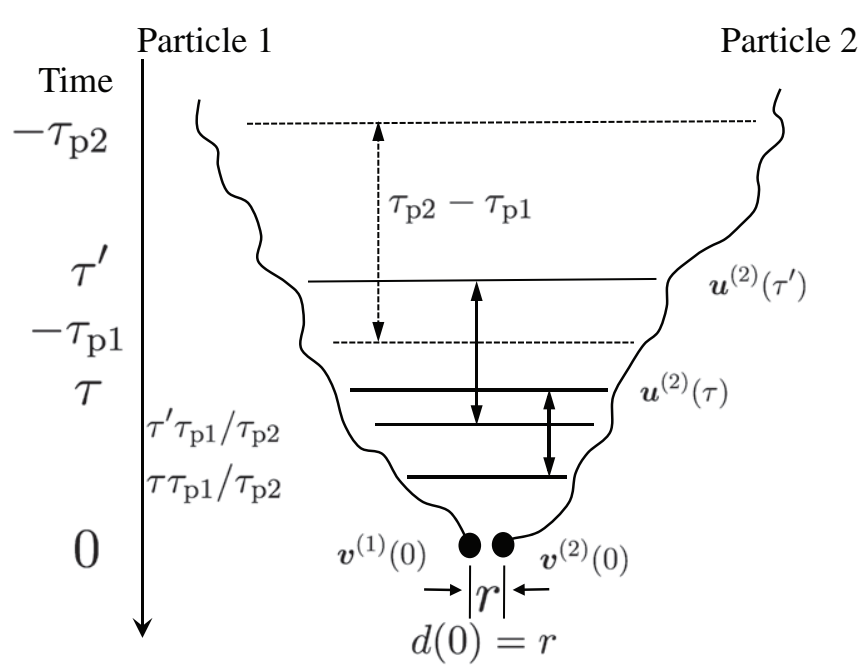

Figure 1. Schematic figure illustrating the generalized acceleration term, $\mathcal{A}_{i j}$, for the relative velocity of particles 1 and 2 of different sizes at a separation $r$ at $t=0$. Without loss of generality, it is assumed $\tau_{\mathrm{p} 2}>\tau_{\mathrm{p} 1}$. The figure is based on Equation (15), which suggests that the effect of $\mathcal{A}_{i j}$ can be roughly viewed as due to the particle memory of the temporal flow velocity difference, $\Delta_{\mathrm{T}} \boldsymbol{u}$, seen by particle $2 . \Delta_{\mathrm{T}} \boldsymbol{u}$ is thus crucial for understanding the generalized acceleration effect.

along the trajectory of particle 2 only. ${ }^{6}$ If $\tau_{\mathrm{p} 1}=0$, then $f=$ 0, and Equation (15) becomes identical to Equation (4) for the flow-particle relative velocity. Based on Equation (15), Figure 1 illustrates a physical picture for $\mathcal{A}_{i j}$. The ensemble average term in Equation (15) is expected to increase with $|\tau|$ and $\left|\tau^{\prime}\right|$, and together with the exponential cutoffs, it suggests that a major contribution to the integral is likely from $\tau$ and $\tau^{\prime}$ values around $-\tau_{\mathrm{p} 2}$. Therefore, a rough estimate for the generalized acceleration effect is $\boldsymbol{w}_{\mathrm{a}} \sim \boldsymbol{u}^{(2)}\left(-\tau_{\mathrm{p} 2}\right)-\boldsymbol{u}^{(2)}\left(-\tau_{\mathrm{p} 1}\right)$, which is essentially the temporal flow velocity difference $\Delta \boldsymbol{u}_{\mathrm{T}}(\Delta \tau)$ along the trajectory of particle 2 at a time lag of $\Delta \tau \simeq\left|\tau_{\mathrm{p} 2}-\tau_{\mathrm{p} 1}\right|$. This establishes a relation between $\boldsymbol{w}_{\mathrm{a}}$ and the temporal statistics of the flow velocity along an individual trajectory. The approximation $\boldsymbol{w}_{\mathrm{a}} \simeq \Delta \boldsymbol{u}_{\mathrm{T}}\left(\left|\tau_{\mathrm{p} 2}-\tau_{\mathrm{p} 1}\right|\right)$ is crude especially for particles of similar size and for large particles with $\tau_{\mathrm{p}} \gtrsim T_{\mathrm{L}}$. We will give a better expression for $\boldsymbol{w}_{\mathrm{a}}$ based on a quantitative calculation of $\mathcal{A}_{i j}$ (see Equation (21) below). Although the discussion here is qualitative, it does provide an insightful physical picture for the generalized acceleration effect. The discussion also suggests that, even if the trajectory statistics of two different particles were identical at all times in the past, their velocities at the current time would be different, as they have different memories of (or different responses to) the flow velocity.

We now quantitatively evaluate the generalized acceleration term. Assuming $B_{i j}^{(1,2)}\left(\tau, \tau^{\prime}\right)=B_{i j}^{(1,2)}\left(\left|\tau^{\prime}-\tau\right|\right)$, we can simplify the double integrals in Equation (A6) by a variable change, yielding

$$
\begin{aligned}
\mathcal{A}_{i j}= & \int_{-\infty}^{0}\left[\left(\frac{\tau_{\mathrm{p} 2}-\tau_{\mathrm{p} 1}}{\tau_{\mathrm{p} 1}+\tau_{\mathrm{p} 2}}\right) B_{i j}^{+}(\Delta \tau)+B_{i j}^{-}(\Delta \tau)\right] \\
& \times\left[\frac{1}{\tau_{\mathrm{p} 1}} \exp \left(\frac{\Delta \tau}{\tau_{\mathrm{p} 1}}\right)-\frac{1}{\tau_{\mathrm{p} 2}} \exp \left(\frac{\Delta \tau}{\tau_{\mathrm{p} 2}}\right)\right] d \Delta \tau,
\end{aligned}
$$

\footnotetext{
6 One could write an approximate equation for $\mathcal{A}_{i j}$ with $\boldsymbol{u}^{(1)}$ only. It would be similar to Equation (15), but with $\boldsymbol{u}^{(1)}$ replacing $\boldsymbol{u}^{(2)}, \tau_{\mathrm{p} 1}$ replacing $\tau_{\mathrm{p} 2}$, and $1 / f$ replacing $f$.
}

where $B_{i j}^{+} \equiv\left(B_{i j}^{(1)}+B_{i j}^{(2)}\right) / 2$ and $B_{i j}^{-} \equiv\left(B_{i j}^{(1)}-B_{i j}^{(2)}\right) / 2$. Using $D_{i j}^{(1,2)}=2\left(u^{\prime 2} \delta_{i j}-B_{i j}^{(1,2)}\right)$ for the temporal flow structure function, $D_{i j}^{(1,2)}$ (see definition below Equation 5), we can explicitly see that the acceleration term is connected to the temporal flow velocity difference, $\Delta_{\mathrm{T}} \boldsymbol{u}(\Delta \tau)$, on the particle trajectories.

Following PP10, we approximate both $B_{i j}^{(1)}$ and $B_{i j}^{(2)}$ by the Lagrangian correlation tensor, $u^{\prime 2} \Phi_{\mathrm{L}}(\Delta \tau) \delta_{i j}$, so that $B_{i j}^{+}=$ $u^{\prime 2} \Phi_{\mathrm{L}}(\Delta \tau) \delta_{i j}$ and $B_{i j}^{-}=0$. Here, the possible asymmetry (i.e., $B_{i j}^{-}$) between the trajectory statistics of different particles is neglected. Equation (16) then becomes

$$
\mathcal{A}_{i j}=A \delta_{i j}
$$

with

$$
\begin{aligned}
A= & u^{\prime 2}\left(\frac{\tau_{\mathrm{p} 2}-\tau_{\mathrm{p} 1}}{\tau_{\mathrm{p} 1}+\tau_{\mathrm{p} 2}}\right) \int_{-\infty}^{0} \Phi_{\mathrm{L}}(\Delta \tau) \\
& \times\left[\frac{1}{\tau_{\mathrm{p} 1}} \exp \left(\frac{\Delta \tau}{\tau_{\mathrm{p} 1}}\right)-\frac{1}{\tau_{\mathrm{p} 2}} \exp \left(\frac{\Delta \tau}{\tau_{\mathrm{p} 2}}\right)\right] d \Delta \tau .
\end{aligned}
$$

Equation (17) indicates that $\mathcal{A}_{i j}$ has equal longitudinal (radial) and transverse (tangential) components.

If we adopt an exponential form for $\Phi_{\mathrm{L}}\left(\equiv \exp \left(-|\Delta \tau| / T_{\mathrm{L}}\right)\right)$, then a simple integration gives

$$
A=u^{\prime 2}\left(\Omega_{2}-\Omega_{1}\right)^{2} /\left[\left(\Omega_{1}+\Omega_{2}\right)\left(1+\Omega_{1}\right)\left(1+\Omega_{2}\right)\right],
$$

where $\Omega_{1,2} \equiv \tau_{\mathrm{p} 1,2} / T_{\mathrm{L}}$. With a bi-exponential $\Phi(\Delta \tau)$ (Equation (7)), one obtains

$$
A=u^{\prime 2} \frac{\left(\Omega_{2}-\Omega_{1}\right)^{2}\left(\Omega_{1} \Omega_{2}+\left(\Omega_{1}+\Omega_{2}\right) \frac{z^{2}}{2}\right)}{\left(\Omega_{1}+\Omega_{2}\right)\left(\Omega_{1}+\Omega_{1}^{2}+\frac{z^{2}}{2}\right)\left(\Omega_{2}+\Omega_{2}^{2}+\frac{z^{2}}{2}\right)},
$$

which reduces to Equation (19) if $z=0$. Clearly, if both $\Omega_{1}$ and $\Omega_{2}$ are much larger than $z^{2} / 2$, Equation (20) is a good approximation for Equation (19). In fact, Equations (19) and (20) are close to each other if either of the two $\Omega$ s is much larger than $z^{2} / 2$. A numerical comparison of Equations (19) and (20) shows that if the large particle has $\Omega \gtrsim 3 z^{2}$, the difference in the two equations is $\lesssim 20 \%$. For convenience, we will denote $\Omega$ of the larger and smaller particles as $\Omega_{h}$ and $\Omega_{\ell}$, respectively. In the limit $\Omega_{h} \rightarrow \infty$, Equation (20) approaches $u^{\prime 2}\left(\Omega_{\ell}+z^{2} / 2\right) /\left(\Omega_{\ell}+\Omega_{\ell}^{2}+z^{2} / 2\right)$, which is the one-particle velocity variance of the smaller particle (see Equation (6) of Paper I).

For small particles with $\Omega_{1,2} \ll z^{2} / 2$, it is easy to show that Equation (20) reduces to $A=2 u^{\prime 2}\left(\Omega_{2}-\Omega_{1}\right)^{2} / z^{2}=\left(\tau_{\mathrm{p} 2}-\right.$ $\left.\tau_{\mathrm{p} 1}\right)^{2} a^{2}$, meaning that the acceleration term in the S-T formula, Equation (12), is correctly reproduced by Equation (20). On the other hand, Equation (19) from the single-exponential $\Phi_{\mathrm{L}}$ fails to recover the $\mathrm{S}-\mathrm{T}$ acceleration term, suggesting that the bi-exponential form (Equation (7)) that accounts for the effect of the flow acceleration is a preferred choice for small particles. A numerical comparison with Equation (20) shows that the S-T acceleration term is valid only if both particles are quite small with $\Omega \lesssim 0.08 z^{2}$, and it becomes inaccurate if $\Omega_{h} \gtrsim 0.08 z^{2}$.

In a short summary, if the larger particle has $\Omega_{h} \lesssim 0.08 z^{2}$, the $\mathrm{S}$-T formula applies, and the acceleration effect is completely determined by the local flow acceleration, $a$. If $\Omega_{h} \gtrsim 3 z^{2}$, the 


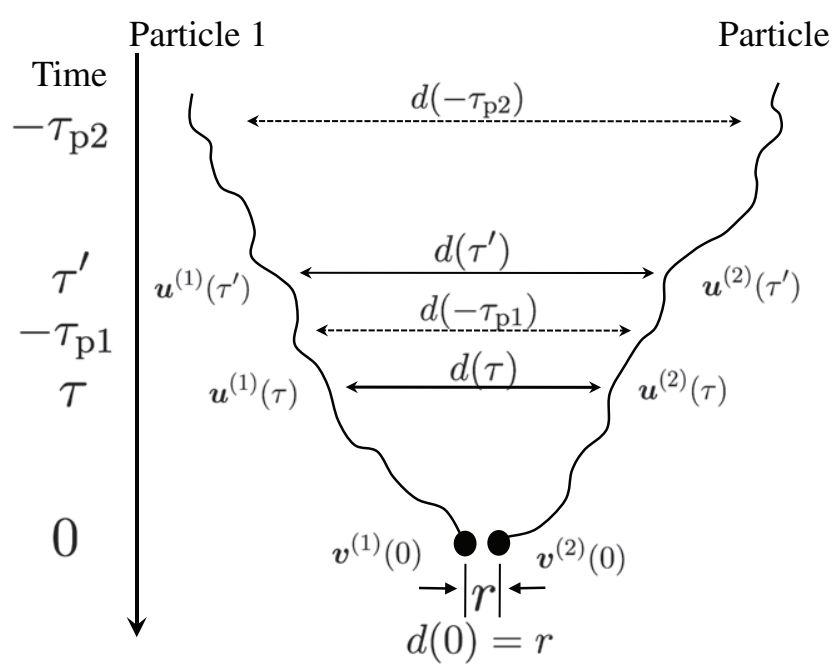

Figure 2. Schematic figure illustrating the generalized shear term for the relative velocity of two different particles, 1 and 2 , which represents the memory of the spatial flow velocity difference "seen" by the two particles in the past. It depends on the particle separations, $d(\tau)$ and $d\left(\tau^{\prime}\right)$, backward in time and also on the temporal decorrelation of the flow velocity structures across the particle distance between $\tau$ and $\tau^{\prime}$.

generalized acceleration term is insensitive to $a$, and one can estimate $A$ using either Equation (19) or Equation (20). In the intermediate case with $0.08 z^{2} \lesssim \Omega_{h} \lesssim 3 z^{2}$, we need to use the general formula, Equation (20), for an accurate estimate. In this case, $A$ depends on the temporal flow velocity difference at a time lag in between the dissipation and inertial ranges of the flow.

If one of the particles, e.g., particle 1 , is a tracer particle, i.e., $\Omega_{1}=0$, then Equation (20) becomes $A=u^{\prime 2} \Omega_{2}^{2} /\left(\Omega_{2}+\Omega_{2}^{2}+\right.$ $\left.z^{2} / 2\right)$. It is identical to the flow-particle relative velocity, $\boldsymbol{w}_{\mathrm{f}}$, derived in Section 2, which is a special bidisperse case where only the generalized acceleration term contributes.

Using Equation (20), we attempted to establish an approximate relation for $\boldsymbol{w}_{\mathrm{a}}$ with the temporal flow velocity difference, $\Delta \boldsymbol{u}_{\mathrm{T}}$, along the particle trajectory. The idea is to obtain an approximate expression for $\boldsymbol{w}_{\mathrm{a}}$ in terms of $\Delta \boldsymbol{u}_{\mathrm{T}}$, which satisfies the condition that the variance of $\boldsymbol{w}_{\mathrm{a}}$ is consistent with Equation (20). A good approximation is found to be

$$
\boldsymbol{w}_{\mathrm{a}} \simeq \frac{1-f}{\left(1+\Omega_{\ell}\right)^{1 / 2}} \Delta \boldsymbol{u}_{\mathrm{T}}\left(\tau_{\mathrm{p}, \mathrm{h}}\right),
$$

where $\tau_{\mathrm{p}, 1}=\min \left(\tau_{\mathrm{p} 1}, \tau_{\mathrm{p} 2}\right), \tau_{\mathrm{p}, \mathrm{h}}=\max \left(\tau_{\mathrm{p} 1}, \tau_{\mathrm{p} 2}\right), \Omega_{\ell, h}=$ $\tau_{\mathrm{p}, 1, \mathrm{~h}} / T_{\mathrm{L}}$, and $f=\tau_{\mathrm{p}, 1} / \tau_{\mathrm{p}, \mathrm{h}}$. We computed the variance of $\boldsymbol{w}_{\mathrm{a}}$ from the above equation under the assumption that $\Delta \boldsymbol{u}_{\mathrm{T}} \simeq \Delta \boldsymbol{u}_{\mathrm{L}}$ and found that it agrees with Equation (20) within a factor of two for $\tau_{\mathrm{p} 1}$ and $\tau_{\mathrm{p} 2}$ in any range. Note that here $\boldsymbol{w}_{\mathrm{a}}$ is related to $\Delta \boldsymbol{u}_{\mathrm{T}}$ at a time lag equal to the friction time, $\tau_{\mathrm{p}, \mathrm{h}}$, of the larger particle. Equation (21) is useful for understanding our simulation result for the bidisperse relative velocity (see Section 6).

\subsubsection{The Generalized Shear Term}

The generalized shear term is given by Equation (A7) in Appendix A, and it represents the contribution to the relative velocity from the particles' memory of the spatial flow velocity differences along their trajectories in the past. The physical picture is illustrated in Figure 2, which is similar to Figure 1 of Paper I for identical particles, except that here the memory cutoffs occur at different times. The key in the PP10 model for this term is the evaluation of the trajectory structure tensor, $S_{\mathrm{T} i j} \equiv\left\langle\left[u_{i}^{(1)}(\tau)-u_{i}^{(2)}(\tau)\right]\left[u_{j}^{(1)}\left(\tau^{\prime}\right)-u_{j}^{(2)}\left(\tau^{\prime}\right)\right]\right\rangle$, defined as the correlation of the flow velocity differences the particles saw at two times, $\tau$ and $\tau^{\prime}$ (Equation (A4) in Appendix A). We modeled it as

$$
S_{\mathrm{T} i j}\left(\boldsymbol{r} ; \tau, \tau^{\prime}\right) \simeq\left\langle S_{i j}(\boldsymbol{R}) \Phi_{2}\left(\tau^{\prime}-\tau, R\right)\right\rangle_{\boldsymbol{R}},
$$

where $\boldsymbol{R}\left(\tau, \tau^{\prime}\right)$ is the typical particle separation between $\tau$ and $\tau^{\prime}$. The ensemble average is over the probability distribution of the random vector $\boldsymbol{R}$. The Eulerian structure tensor $S_{i j}(\boldsymbol{R})$ is defined as $\left\langle\Delta u_{i}(\boldsymbol{R}) \Delta u_{j}(\boldsymbol{R})\right\rangle$, with $\Delta \boldsymbol{u}(\boldsymbol{R}) \equiv \boldsymbol{u}(\boldsymbol{x}+\boldsymbol{R})-\boldsymbol{u}(\boldsymbol{x})$, and $\Phi_{2}\left(\tau^{\prime}-\tau, R\right)$ is the temporal (de)correlation of the flow velocity structures at a time lag of $\Delta \tau=\tau^{\prime}-\tau$. A simple assumption of $\Phi_{2}$ is $\left.\Phi_{2} \simeq \exp \left[-\left|\tau^{\prime}-\tau\right|\right) / T(R)\right]$, where $T(R)$ is correlation time (or lifetime) of turbulent eddies of size $R$. A better approximation for $\Phi_{2}$ with a bi-exponential form is given in Appendix B. Note that $\Phi_{2}$ differs from the temporal correlation, $\Phi_{1}$, on the trajectory of an individual particle.

We neglect the fluctuations in the amplitude, $R$, of $\boldsymbol{R}$ and estimate $S_{\mathrm{T} i j}$ by simply using the rms value of $R$ (PP10). In the rest of the paper, $R$ denotes the rms particle distance. We then have $S_{\mathrm{T} i j}\left(\boldsymbol{r} ; \tau, \tau^{\prime}\right) \simeq\left\langle S_{i j}(\boldsymbol{R})\right\rangle_{\text {ang }} \Phi_{2}\left(\tau^{\prime}-\tau, R\right)$, where "ang" denotes the angular average over the direction of $\boldsymbol{R}$. Inserting it into Equation (A7) in Appendix A leads to

$$
\begin{aligned}
\mathcal{S}_{i j}= & \int_{-\infty}^{0} \frac{d \tau}{\tau_{\mathrm{p} 1}} \int_{-\infty}^{0} \frac{d \tau^{\prime}}{\tau_{\mathrm{p} 2}}\left\langle S_{i j}(\boldsymbol{R})\right\rangle_{\mathrm{ang}} \Phi_{2}\left(\tau^{\prime}-\tau, R\right) \\
& \times \exp \left(\frac{\tau}{\tau_{\mathrm{p} 1}}\right) \exp \left(\frac{\tau^{\prime}}{\tau_{\mathrm{p} 2}}\right) .
\end{aligned}
$$

An approximate evaluation of $\left\langle S_{i j}(\boldsymbol{R})\right\rangle_{\text {ang }}$ in Appendix B gives

$$
\left\langle S_{i j}(\boldsymbol{R})\right\rangle_{\mathrm{ang}}=\frac{1}{3}\left[S_{\mathrm{ll}}(R)+2 S_{\mathrm{nn}}(R)\right] \delta_{i j},
$$

where $S_{\mathrm{ll}}$ and $S_{\mathrm{nn}}$ are the longitudinal and transverse structure functions of the flow, respectively. The dependence of $S_{\mathrm{T} i j}$ on $R$ indicates the crucial role of the particle separation backward in time. We estimate $R\left(\tau, \tau^{\prime}\right)$ as the geometric average of the particle distances, $d(\tau)$ and $d\left(\tau^{\prime}\right)$, at $\tau$ and $\tau^{\prime}$, i.e., $R\left(\tau, \tau^{\prime}\right)=$ $\sqrt{d(\tau) d\left(\tau^{\prime}\right)}$. A two-phase separation behavior consisting of a ballistic phase $\left(d^{2}(\tau)=r^{2}+\left\langle w^{2}\right\rangle \tau^{2}\right.$ with $\left\langle w^{2}\right\rangle$ the 3D variance of the particle relative velocity) and a Richardson phase $\left(d(\tau)^{2} \propto g \bar{\epsilon}|\tau|^{3}\right.$ with $g$ the Richardson constant) is used for $d(\tau)$ as a function of $\tau$ (Paper I). We connect the two behaviors at a transition time, $\tau_{\mathrm{c}}=-\left(\tau_{\mathrm{p} 1}+\tau_{\mathrm{p} 2}\right) / 2$. The justification for the assumptions is described in Appendix B.

Based on Equation (23), we give an approximate estimate for the shear term $\boldsymbol{w}_{\mathrm{s}}$ and relate it to the spatial flow velocity difference, $\Delta \boldsymbol{u}(\ell)(\simeq u(x+\ell)-u(x))$. As shown in Section 3.24 of Paper I, the shear term can be written as $\boldsymbol{w}_{\mathrm{s}} \simeq$ $\Delta \boldsymbol{u}\left(R_{\mathrm{p}}\right)\left[T_{\mathrm{p}} /\left(T_{\mathrm{p}}+\tau_{\mathrm{p}}\right)\right]^{1 / 2}$ for equal-size particles with a friction time $\tau_{\mathrm{p}}$. Here $R_{\mathrm{p}}$, named the primary distance in PP10 and Paper I, is defined as $R_{\mathrm{p}} \equiv R\left(-\tau_{\mathrm{p}},-\tau_{\mathrm{p}}\right)$, which is of particular interest because the memory cutoff sets in at $\tau, \tau^{\prime} \lesssim-\tau_{\mathrm{p}}$. In Paper I, we set $R_{\mathrm{p}} \simeq\left\langle w^{2}\right\rangle^{1 / 2} \tau_{\mathrm{p}}$, which assumes the duration of the ballistic separation phase is not shorter than $\tau_{\mathrm{p}}$. The timescale $T_{\mathrm{p}}$ is the flow correlation (or eddy turnover) time at the scale $R_{\mathrm{p}}$, i.e., $T_{\mathrm{p}}=T\left(R_{\mathrm{p}}\right)$, and the factor $\left[T_{\mathrm{p}} /\left(T_{\mathrm{p}}+\tau_{\mathrm{p}}\right)\right]^{1 / 2}$ is due to the $\Phi_{2}$ term in Equation (23) that gives a constraint, $\left|\tau^{\prime}-\tau\right| \lesssim T(R)$, on the memories of the two particles that can contribute (Paper I).

In the bidisperse case, an expression for $\boldsymbol{w}_{\mathrm{s}}$ is more complicated due to the different friction times. In this case, the $\Phi_{2}$ term 
tends to limit or reduce the temporal range of the large particle's memory (around the memory time of the smaller particle) that can contribute to $\boldsymbol{w}_{\mathrm{s}}$. Roughly speaking, $R_{\mathrm{p}}$ is primarily determined by the smaller particle, and a simple assumption ${ }^{7}$ would be $R_{\mathrm{p}} \simeq R\left(-\tau_{\mathrm{p}, 1},-\tau_{\mathrm{p}, 1}\right)$, where $\tau_{\mathrm{p}, 1}$ is the friction time of the smaller particle. Analogous to the monodisperse case, we then have

$$
\boldsymbol{w}_{\mathrm{s}} \simeq \Delta \boldsymbol{u}\left(R_{\mathrm{p}}\right)\left(\frac{T_{\mathrm{p}}}{T_{\mathrm{p}}+\tau_{\mathrm{p}, \mathrm{h}}}\right)^{1 / 2}
$$

where the last term corresponds to the reduction in the time range of the larger particle's memory that can contribute when $T_{\mathrm{p}}<\tau_{\mathrm{p}, \mathrm{h}}$. Although the assumption above is rough, it provides a useful picture to understand the generalized shear term.

\subsection{Summary}

We briefly summarize our model for the general bidisperse case. Using Equations (17), (23), and (24), the 3D rms relative velocity can be calculated from

$$
\left\langle w^{2}\right\rangle=3 A+\mathcal{S}_{i i},
$$

where $A$ is given by Equation (17) and

$$
\begin{aligned}
\mathcal{S}_{i i}= & \int_{-\infty}^{0} \frac{d \tau}{\tau_{\mathrm{p} 1}} \int_{-\infty}^{0} \frac{d \tau^{\prime}}{\tau_{\mathrm{p} 2}}\left[S_{\mathrm{ll}}(R)+2 S_{\mathrm{nn}}(R)\right] \Phi_{2}\left(\tau^{\prime}-\tau, R\right) \\
& \times \exp \left(\frac{\tau}{\tau_{\mathrm{p} 1}}\right) \exp \left(\frac{\tau^{\prime}}{\tau_{\mathrm{p} 2}}\right)
\end{aligned}
$$

Here $S_{11}, S_{\mathrm{nn}}$, and the timescale $T$ in $\Phi_{2}$ are given by Equations (B6), (B7), and (B8) in Appendix B. Equation (26) is an implicit equation of $\left\langle w^{2}\right\rangle$, as $R$ in Equation (27) depends on $\left\langle w^{2}\right\rangle$ in the ballistic separation phase (see Section 3.2.2 and Equation (B4) in Appendix B). We solve Equation (26) by an iteration method. Unlike the monodisperse case of equal-size particles, the particle separation rate in the ballistic phase (which is given by $\left\langle w^{2}\right\rangle^{1 / 2}$ ) has a contribution from the generalized acceleration term.

The generalized acceleration and shear terms can recover the $\mathrm{S}$-T prediction in the small-particle limit with $\mathrm{St}_{1,2} \ll 1$ (see Section 3.2.1 and Appendices A and B). Here we show that Equation (26) also correctly reproduces the prediction, Equation (13), for the large-particle limit, i.e., $\tau_{\mathrm{p} 1, \mathrm{p} 2} \gg T_{\mathrm{L}}$ (or $\Omega_{1,2} \gg 1$ ). We start with an analysis of the generalized shear term, i.e., Equation (27). We first note that $S_{\mathrm{ll}}(R), S_{\mathrm{nn}}(R)$, and $T(R)$ increase as $R$ increases backward in time toward the integral scale, $L$, of the turbulent flow. At $|\tau|,\left|\tau^{\prime}\right| \gtrsim T_{\mathrm{L}}, R$ is expected to exceed $L$ (see Paper I), and $S_{11}(R), S_{\mathrm{nn}}(R)$, and $T(R)$ become constant, i.e., $S_{11}(R)=$ $S_{\mathrm{nn}}(R)=2 u^{\prime 2}$, and $T(R)=T_{\mathrm{L}}$ (see Equations (B6), (B7), and (B8)). We thus have $\left[S_{11}(R)+2 S_{\mathrm{nn}}(R)\right] \Phi_{2}\left(\tau-\tau^{\prime}, R\right)=$ $6 u^{\prime 2} \Phi_{\mathrm{L}}\left(\tau-\tau^{\prime}\right)$ for $\tau, \tau^{\prime} \lesssim-T_{\mathrm{L}}$. If the cutoff timescales, $\tau_{\mathrm{p} 1}$ and $\tau_{\mathrm{p} 2}$, in Equation (27) are much larger than $T_{\mathrm{L}}$, one may approximate $\mathcal{S}_{i i}$ by $\simeq 6 u^{\prime 2} \int_{-\infty}^{0}\left(d \tau / \tau_{\mathrm{p} 1}\right) \int_{-\infty}^{0}\left(d \tau^{\prime} / \tau_{\mathrm{p} 2}\right) \Phi_{\mathrm{L}}(\tau-$ $\left.\tau^{\prime}\right) \exp \left(\tau / \tau_{\mathrm{p} 1}\right) \exp \left(\tau^{\prime} / \tau_{\mathrm{p} 2}\right)$, which approaches $12 u^{\prime 2} /\left(\Omega_{1}+\Omega_{2}\right)$

\footnotetext{
$7 \quad$ A more accurate evaluation of $R_{\mathrm{p}}$ can be obtained as follows. One may first compute $R_{1, \mathrm{~h}}=R\left(-\tau_{\mathrm{p}, \mathrm{l}},-\tau_{\mathrm{p}, \mathrm{h}}\right)$, with $\tau_{\mathrm{p}, \mathrm{l}, \mathrm{h}}$ the friction times of smaller and larger particles, and then compare $T\left(R_{1, \mathrm{~h}}\right)$ with $\tau_{\mathrm{p}, \mathrm{h}}-\tau_{\mathrm{p}, \mathrm{l}}$. If $T\left(R_{\mathrm{l}, \mathrm{h}}\right)$ is larger, we set $R_{\mathrm{p}}=R_{\mathrm{l}, \mathrm{h}}$ and $T_{\mathrm{p}}=T\left(R_{\mathrm{l}, \mathrm{h}}\right)$. Otherwise, we define $R_{\mathrm{p}}$ such that $R_{\mathrm{p}}=R\left(-\tau_{\mathrm{p}, 1},-\tau_{\mathrm{p}, 1}-T_{\mathrm{p}}\right)$. Combining this with $T_{\mathrm{p}}=T\left(R_{\mathrm{p}}\right)$, one can solve $R_{\mathrm{p}}$ and $T_{\mathrm{p}}$. Using these estimates of $R_{\mathrm{p}}$ and $T_{\mathrm{p}}$, we find that the variance of Equation (25) provides a satisfactory approximation for the shear contribution, $\mathcal{S}_{i j}$.
}

in the limit $\Omega_{1,2} \gg 1$. Since $3 A=3 u^{\prime 2}\left(\Omega_{1}-\Omega_{2}\right)^{2} /\left[\Omega_{1} \Omega_{2}\left(\Omega_{1}+\right.\right.$ $\left.\left.\Omega_{2}\right)\right]$ at $\Omega_{1,2} \gg 1$, we have $\left\langle w^{2}\right\rangle=3 A+\mathcal{S}_{i i} \rightarrow 3 u^{\prime 2}\left(1 / \Omega_{1}+\right.$ $1 / \Omega_{2}$ ), consistent with Equation (13) in Section 3.1. This proves that the large-particle limit is correctly recovered in our model.

\section{NUMERICAL SIMULATION}

We use the same simulation data as in Paper I. We briefly describe the simulation and refer the reader to Paper I for details. Using the Pencil code, we carried out the simulation in a periodic $512^{3}$ box with a length of $2 \pi$ on each side. An isothermal equation of state was adopted. The flow was driven and maintained by a large-scale force using wave numbers $1 \leqslant k \leqslant 2$. After reaching steady state, the $1 \mathrm{D}$ rms velocity of the flow is $u^{\prime} \simeq 0.05$ in units of the sound speed. The $3 \mathrm{D} \mathrm{rms}$ velocity of the flow corresponds to a Mach number of 0.085 , suitable for applications to protoplanetary disks. At a Mach number $\simeq 0.1$, the compressibility is very low, and the flow statistics of all orders are essentially identical to incompressible turbulence (Pan \& Scannapieco 2011). The integral length, $L$, of the flow is about $1 / 6$ the box size, while the Kolmogorov length scale $\eta \equiv\left(v^{3} / \bar{\epsilon}\right)^{1 / 4}$ is estimated to be 0.6 the cell size of the grid, so that $L \simeq 140 \eta$. The Taylor Reynolds number of the flow is $\operatorname{Re}_{\lambda} \simeq 200$, and the regular Reynolds number is $\mathrm{Re} \simeq 1000$. The Kolmogorov velocity, $u_{\eta} \equiv(\nu \bar{\epsilon})^{1 / 4}$, is related to the rms by $u^{\prime}=\left(\operatorname{Re}_{\lambda} / \sqrt{15}\right)^{1 / 2} u_{\eta} \simeq 7 u_{\eta}$.

A computation of the large eddy turnover time, $T_{\text {eddy }} \simeq$ $L / u^{\prime}$, and the Kolmogorov time, $\tau_{\eta} \equiv(v / \bar{\epsilon})^{1 / 2}$, shows that $T_{\text {eddy }} \simeq 19.2 \tau_{\eta}$. By integrating trajectories of tracer particles $(\mathrm{St}=0)$, we measured the Lagrangian correlation function, $\Phi_{\mathrm{L}}$, which is well fit by the bi-exponential form, Equation (7), with $T_{\mathrm{L}}=14.4 \tau_{\eta}$ and $z=0.3$ (Paper I). The timescale, $T_{\mathrm{E}}$, for the Eulerian temporal correlation was estimated to be $15.9 \tau_{\eta}$. The near equality of $T_{\mathrm{E}}$ with the Lagrangian correlation time $T_{\mathrm{L}}$ justifies the use of the Lagrangian correlation function for all particles (Paper I). In Appendix C, we show the Lagrangian and Eulerian temporal structure functions, $D_{\mathrm{L}}$ and $D_{\mathrm{E}}$, which are of direct relevance for the particle-flow relative velocity and the generalized acceleration term.

We included 14 species of particles of different sizes in our simulated flow, each containing 33.6 million particles. The friction timescale, $\tau_{\mathrm{p}}$, of the smallest particles is $\simeq 0.1 \tau_{\eta}$, while the largest particles have $\tau_{\mathrm{p}}=54 T_{\mathrm{L}}$, corresponding to $\mathrm{St}=795$. Spanning four orders of magnitude, the $\tau_{\mathrm{p}}$ range covers the entire length-scale range of the simulated flow. When integrating the particle equation of motion (Equation (1)), we interpolated the flow velocity inside computational cells using the triangularshaped cloud (TSC) method (Johansen \& Youdin 2007). An accurate integration of the particle trajectories requires an integration time step smaller than $\tau_{\mathrm{p}}$ by a factor of $\gtrsim 10-20$ (see, e.g., Ayala et al. 2007). The time step in our simulation is about $\simeq 0.01$ Kolmogorov time, which was set by the Courant condition for the flow evolution. The ratio of this time step to the friction time of the smallest particles in our simulation is $\simeq 1 / 10$, close to the required value for accurate trajectory integration. Our simulation run lasted $26 T_{\text {eddy }}$, and at the end of the run, all the statistical measures reached a quasi steady state, and the dynamics of all particles was relaxed (Paper I). The simulation was computationally intensive. Using 4096 cores on the NASA/ Ames Pleiades supercomputer, the run lasted 14 days, costing 1.4 million CPU hours. In our data analysis, we use several well-separated snapshots toward the end of the simulation. 


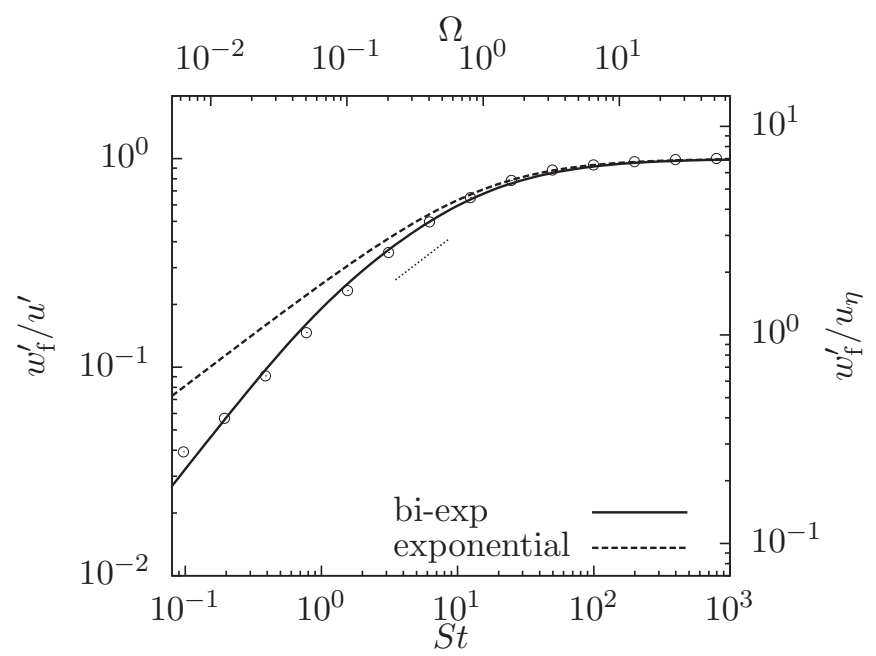

Figure 3. 1D rms of the particle-flow relative velocity as a function of the particle Stokes number, St. The top $x$ axis normalizes $\tau_{\mathrm{p}}$ to the Lagrangian correlation time, $T_{\mathrm{L}}$. The dashed and solid lines are model predictions, Equations (6) and (8), using single- and bi-exponential forms for $\Phi_{\mathrm{L}}$ (and hence for $\Phi_{1}$ ), respectively. In both lines, $T_{\mathrm{L}}$ is taken to be $14.4 \tau_{\eta}$. For the bi-exponential case, the parameter $z$ is set to 0.3 . The dotted line segment denotes a $\mathrm{St}^{1 / 2}$ scaling.

\section{THE PARTICLE-FLOW RELATIVE VELOCITY}

In Figure 3, we show the simulation results for the 1D rms of the particle-flow relative velocity, $\boldsymbol{w}_{\mathrm{f}}$. To compute $\boldsymbol{w}_{\mathrm{f}}$, we interpolated the flow velocity at the position of each particle with the same TSC method used in the simulation. The computed relative velocity is for zero particle-flow distance. The bottom and top axes normalize the particle friction time to the Kolmogorov and Lagrangian correlation times, respectively. The rms relative velocity, $w_{\mathrm{f}}^{\prime}$, is normalized to the flow $\mathrm{rms}\left(u^{\prime}\right)$ and the Kolmogorov velocity $\left(u_{\eta}\right)$ on the left and right $y$ axes, respectively. Similar normalizations are used in most figures in the rest of the paper.

Equation (5) from our model suggests that $\boldsymbol{w}_{\mathrm{f}}$ is estimated by the temporal flow velocity difference $\Delta_{\mathrm{T}} \boldsymbol{u}$ along the particle trajectory at a time lag of $\tau_{\mathrm{p}} . \Delta_{\mathrm{T}} \boldsymbol{u}$ may be approximated by the Lagrangian $\left(\Delta_{\mathrm{L}} \boldsymbol{u}\right)$ and Eulerian $\left(\Delta_{\mathrm{E}} \boldsymbol{u}\right)$ temporal velocity differences for particles in the St $\ll 1$ and $\tau_{\mathrm{p}} \gg T_{\mathrm{L}}$ limits, respectively. For a better understanding of $\boldsymbol{w}_{\mathrm{f}}$, we show the Lagrangian and Eulerian temporal structure functions $\left(D_{\mathrm{L}}\right.$ and $D_{\mathrm{E}}$ ) in Figure 11 of Appendix C. As argued in Appendix C, the behaviors of $\Delta_{\mathrm{L}} \boldsymbol{u}$ and $\Delta_{\mathrm{E}} \boldsymbol{u}$ suggest that one may approximate $\Delta_{\mathrm{T}} \boldsymbol{u}$ by the Lagrangian velocity difference for particles of any size. Comparing with Figure 11 in Appendix C, we see that $w_{f}^{\prime}$ as a function of St is similar to the Lagrangian velocity difference as a function of the time lag, confirming the physical picture of our model.

The solid line in Figure 3 is our model prediction, Equation (8), using a bi-exponential form for the Lagrangian correlation function $\Phi_{\mathrm{L}}$ (and hence for $\Phi_{1}$; see Section 2). In $\Phi_{\mathrm{L}}$, we set $T_{\mathrm{L}}$ to $14.4 \tau_{\eta}$ and the parameter $z$ to 0.3 . These values of $T_{\mathrm{L}}$ and $z$ were obtained from the best fit of Equation (7) to the Lagrangian correlation function measured in our simulated flow (see Figure 2 of Paper I and Figure 11 in Appendix C of the current paper). As mentioned in Section 2, the parameter $z$ has a dependence on the flow Reynolds number, Re, and, considering $\operatorname{Re} \simeq 10^{3}$ in our flow, one can estimate $z$ in protoplanetary turbulence using appropriate values of $\mathrm{Re}$ and the scaling $z \simeq 0.3\left(\operatorname{Re} / 10^{3}\right)^{-1 / 4}$. The solid line fits the data well except for the smallest particles.
The dashed line corresponds to Equation (6) based on a singleexponential $\Phi_{\mathrm{L}}$. Here $T_{\mathrm{L}}$ is also set to $14.4 \tau_{\eta}$. The fitting quality of Equation (6) cannot be improved by tuning $T_{\mathrm{L}}$. As discussed in Section 2, Equation (6) predicts a $\mathrm{St}^{1 / 2}$ scaling for $w_{\mathrm{f}}^{\prime}$ for all particles with $\Omega \ll 1$. This prediction is inaccurate, and the scaling of $w_{\mathrm{f}}^{\prime}$ is significantly steeper than $\mathrm{St}^{1 / 2}$ at $\mathrm{St} \lesssim 3$. This discrepancy is due to the fact that the single-exponential form does not reflect the smooth part of $\Phi_{\mathrm{L}}$ (or $D_{\mathrm{L}}$ ) at time lags, $\Delta \tau$, below the Taylor microtimescale, $\tau_{\mathrm{T}}$. At $\Delta \tau<\tau_{\mathrm{T}}$, $\Phi_{\mathrm{L}}$ is affected by the flow acceleration. The Taylor timescale was found to be $4.3 \tau_{\eta}$ in our flow (Paper I), and this explains the deviation of the dotted line from the data points at St $\lesssim 3$. Again, the steepening of $w_{\mathrm{f}}^{\prime}$ below $\tau_{\mathrm{p}} \lesssim \tau_{\mathrm{T}}$ corresponds to the transition from the inertial-range scaling of the temporal flow velocity difference, $\Delta \boldsymbol{u}_{\mathrm{T}}$, to the dissipation range, which is not captured by the single-exponential correlation.

The $\mathrm{St}^{1 / 2}$ scaling applies only to inertial-range particles. The dotted line segment in Figure 3 represents such a scaling at intermediate $\mathrm{St}$. The data points do not show a clear $\mathrm{St}^{1 / 2}$ range probably due to the limited inertial range of the simulated flow. A confirmation of the predicted $\mathrm{St}^{1 / 2}$ scaling would require larger resolutions. For small particles in the $\mathrm{St} \ll 1$ limit, $w_{\mathrm{f}}^{\prime}$ is predicted to be equal to $a \tau_{\mathrm{p}}$ (see Section 2), suggesting a linear increase of $w_{\mathrm{f}}^{\prime}$ with St. The linear scaling is not observed even at the smallest $\operatorname{St}(=0.1)$ in our simulation. The linear scaling of $w_{\mathrm{f}}^{\prime}$ is based on the assumptions that tiny particles closely follow Lagrangian trajectories and that the Lagrangian structure function, $D_{\mathrm{L}}$, scales with the time lag as $D_{\mathrm{L}} \propto(\Delta \tau)^{2}$ (see Equation (5)). However, the scaling of $D_{\mathrm{L}}$ was found to be shallower than $\propto(\Delta \tau)^{2}$ at $\Delta \tau \gtrsim 0.1 \tau_{\eta}$ (Figure 11 in Appendix C). This explains why the scaling of $w_{\mathrm{f}}^{\prime}$ is slower than $\propto$ St for St $\gtrsim 0.1$. To verify the linear scaling of $w_{\mathrm{f}}^{\prime}$, one needs to include smaller particles with $\mathrm{St} \ll 0.1$. The data point at $\mathrm{St}=0.1$ is significantly larger than our model prediction with a bi-exponential $\Phi_{\mathrm{L}}$, and the reason may be that the trajectory computation for the smallest particles in our simulation is the least accurate. This is because for these small particles the ratio of the simulation time step to the friction time is the largest, $\simeq 1 / 10$, which only marginally meets the requirement for accurate trajectory integration (see Section 4). In order to improve the accuracy for the St $\lesssim 0.1$ particles, we will adopt a smaller time step for the trajectory integration of the smallest particles in future simulations.

\section{THE PARTICLE RELATIVE VELOCITY}

To compute the particle relative velocity, we search pairs of particles from all the different species at given distances, $r=1$, $1 / 2$, and $(1 / 4) \eta$. For each particle 1 , we count particles 2 in a distance shell $[r-d r / 2, r+d r / 2]$. The shell thickness $d r$ $(\ll r)$ is chosen such that the number of particle pairs available is of the order of $\gtrsim 10^{4}$, sufficient for the measurement of the rms relative velocity at an accuracy level of a few percent. On the other hand, at $r \lesssim(1 / 8) \eta$, the measurement accuracy becomes poor due to the limited number of particles available at small distances (Paper I). We thus limit our measurements to $r \gtrsim(1 / 4) \eta$. Future simulations including a much larger number of particles will allow accurate measurements at $r \lesssim(1 / 8) \eta$ and help resolve the collision statistics at smaller scales toward the particle size. We calculate the 3D amplitude, $|\boldsymbol{w}|$, of the relative velocity and its radial component, $w_{\mathrm{r}}=\boldsymbol{w} \cdot \boldsymbol{r} / r$, for each pair and average over all pairs to obtain the rms values, $\left\langle w^{2}\right\rangle^{1 / 2}$ and 
Table 1

Properties of the Simulated Flow and the Model Parameters

\begin{tabular}{lc}
\hline \hline $\operatorname{Re}$ & 1000 \\
$u^{\prime} / u_{\eta}$ & 7 \\
$L / \eta$ & 140 \\
$T_{\text {eddy }} / \tau_{\eta}$ & 19.2 \\
$T_{\mathrm{L}} / \tau_{\eta}$ & 14.4 \\
$\tau_{\mathrm{T}} / \tau_{\eta}$ & 4.3 \\
$z$ & 0.3 \\
$C_{\mathrm{K}}{ }^{\mathrm{a}}$ & 2 \\
$C_{\mathrm{Kn}}{ }^{\mathrm{b}}$ & 2.5 \\
$C_{\mathrm{T}}{ }^{\mathrm{c}}$ & 0.4 \\
$\tau_{\mathrm{c}}{ }^{\mathrm{d}}$ & $-\left(\tau_{\mathrm{p} 1}+\tau_{\mathrm{p} 2}\right) / 2$ \\
$g^{\mathrm{e}}$ & $1.6(r=1 \eta)$ \\
& $1.3\left(r=\frac{1}{2} \eta\right)$ \\
& $1.0\left(r=\frac{1}{4} \eta\right)$
\end{tabular}

Notes.

${ }^{a}$ Used in Equation (B6) for the longitudinal structure function

b Used in Equation (B7) for the transverse structure function.

${ }^{c}$ Used in Equation (B8) for the eddy timescale as a function of length scale.

d Assumed transition time from the ballistic separation to the Richardson behavior.

e Adopted Richardson constants that best fit $\left\langle w^{2}\right\rangle^{1 / 2}$ at different $r$.

$\left\langle w_{\mathrm{r}}^{2}\right\rangle^{1 / 2}$. The rms speed in a tangential direction is calculated as $\left\langle w_{\mathrm{r}}^{2}\right\rangle^{1 / 2}=\left(\left\langle w^{2}\right\rangle-\left\langle w_{\mathrm{r}}^{2}\right\rangle\right)^{1 / 2} / \sqrt{2}$.

In PP10, we showed that our model prediction for the rms relative velocity is in good agreement with the simulation data of Zhou et al. (2001) for the bidisperse case. Here we test the model more systematically using our simulation (at higher resolution than Zhou et al.). To compare with the data, we first fix the Stokes number, $\mathrm{St}_{1}$, of particles 1 and examine the relative velocity as a function of $\mathrm{St}_{2}$. In Section 6.2, we will study the rms relative velocity at fixed Stokes number ratios, $f \equiv \mathrm{St}_{\ell} / \mathrm{St}_{h}$.

In Table 1, we summarize the properties of the simulated flow and the parameters adopted in our model. The measurements and choices of the parameters are presented in detail in Paper I, to which we refer the reader for details. For example, as mentioned earlier, $T_{\mathrm{L}}$ and $z$ are obtained by fitting Equation (7) to the measured Lagrangian correlation function, $\Phi_{\mathrm{L}}$, while $C_{\mathrm{K}}$ and $C_{\mathrm{Kn}}$ are measured from the structure functions of the flow. The choice of $\tau_{\mathrm{c}}$ is explained in Appendix B, and a detailed discussion on the selection of $g$ values at different $r$ is given in Section 6.3.

\subsection{The rms Relative Velocity at Fixed $S t_{1}$}

In Figure 4, we plot the 3D rms relative velocity, $\left\langle w^{2}\right\rangle^{1 / 2}$, for $\mathrm{St}_{1}=0.78$. The data points are the simulation result for a particle distance of $r=1 \eta$. Clearly, $\left\langle w^{2}\right\rangle^{1 / 2}$ shows a dip at $\mathrm{St}_{2}=\mathrm{St}_{1}$. As discussed in PP10, the correlation between the velocities of equal-size particles is stronger than that between different ones. A higher-velocity correlation corresponds to a smaller relative velocity, leading to the formation of a dip at $\mathrm{St}_{2} \simeq \mathrm{St}_{1}$.

The solid red line is the prediction of our model using a biexponential form for both the one-particle and the two-particle temporal trajectory correlations, $\Phi_{1}$ and $\Phi_{2}$. As explained above, we set the Lagrangian correlation timescale to $14.4 \tau_{\eta}$ and the parameter $z$ to 0.3 . For the calculation of the shear contribution,

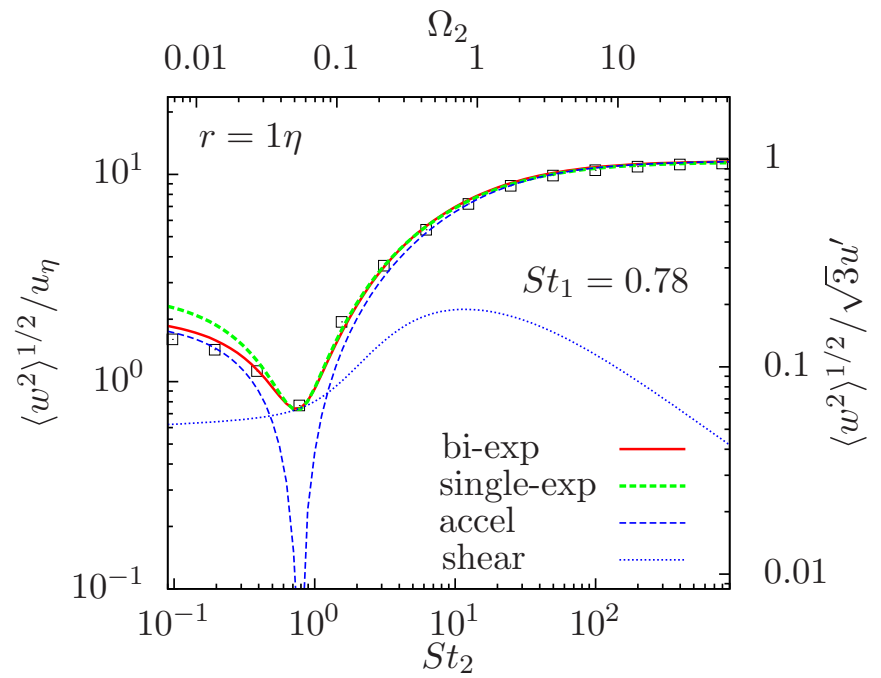

Figure 4. $3 \mathrm{D}$ rms relative velocity as a function of $\mathrm{St}_{2}$ for $\mathrm{St}_{1}=0.78$. Red and green lines are our model predictions using bi-exponential and singleexponential temporal correlation functions $\left(\Phi_{1}\right.$ and $\left.\Phi_{2}\right)$, respectively. A twophase separation is adopted for the generalized shear contribution. The ballistic phase is assumed to last for $\left(\tau_{\mathrm{p} 1}+\tau_{\mathrm{p} 2}\right) / 2$, and $g$ is set to 1.6 for the Richardson phase. The blue dashed and dotted lines correspond to the contributions by the generalized acceleration $(\sqrt{3 A})$ and shear $\left(\sqrt{\mathcal{S}_{i i}}\right)$ terms in our model with bi-exponential temporal correlations.

(A color version of this figure is available in the online journal.)

we adopt a two-phase backward-in-time separation behavior consisting of an initial ballistic phase and a Richardson phase. The motivation and the justification for the assumed behavior can be found in Paper I and Appendix B of the current paper. The ballistic phase is assumed to last for a duration of $\left(\tau_{\mathrm{p} 1}+\tau_{\mathrm{p} 2}\right) / 2$ for particles of different sizes and then connect to a Richardson phase with a Richardson constant of $g=1.6$. The same separation is used in all our model predictions for $r=1 \eta$ in the rest of the paper. When $\mathrm{St}_{2}=\mathrm{St}_{1}$, the assumed separation behavior reduces to that of identical particle pairs discussed in Paper I. The choice of $g=1.6$ is because, as shown in Paper I, it gives a successful fit to the simulation data for the relative velocity of equal-size particles at $r=1 \eta$. As mentioned earlier, a difference for the pair separation of different particles from the case of equal-size particles is that the separation rate in the ballistic phase has a contribution from the generalized acceleration term. The red line is in good agreement with the data, supporting the physical picture of our model for the bidisperse case. The dip center in the red line corresponds to our prediction for the monodisperse case with $\mathrm{St}=0.78$. We point out that the separation behavior we used for particles of different sizes is largely an educated guess. However, the exact behavior turns out to be unimportant for very different particles, as the main contribution to their relative velocity is the generalized acceleration term (see below).

The blue dashed and dotted lines in Figure 4 correspond to the generalized acceleration $(\sqrt{3 A})$ and shear $\left(\sqrt{\mathcal{S}_{i i}}\right)$ terms in our model with bi-exponential temporal correlations. As discussed in Section 3, the acceleration term vanishes for identical particles, and this is responsible for the dip of $\left\langle w^{2}\right\rangle^{1 / 2}$ at $\mathrm{St}_{2} \simeq \mathrm{St}_{1}$. It increases the relative velocity on both sides of the dip. The generalized shear term first increases with $\mathrm{St}_{2}$ and then turns over and decreases at large $\mathrm{St}_{2}$. This can be understood from the approximate Equation (25) for the shear term, $\boldsymbol{w}_{\mathrm{s}}$. The primary distance, $R_{\mathrm{p}}$, in Equation (25) is mainly controlled by the smaller particle, and for $\mathrm{St}_{2}<\mathrm{St}_{1}$, it increases 


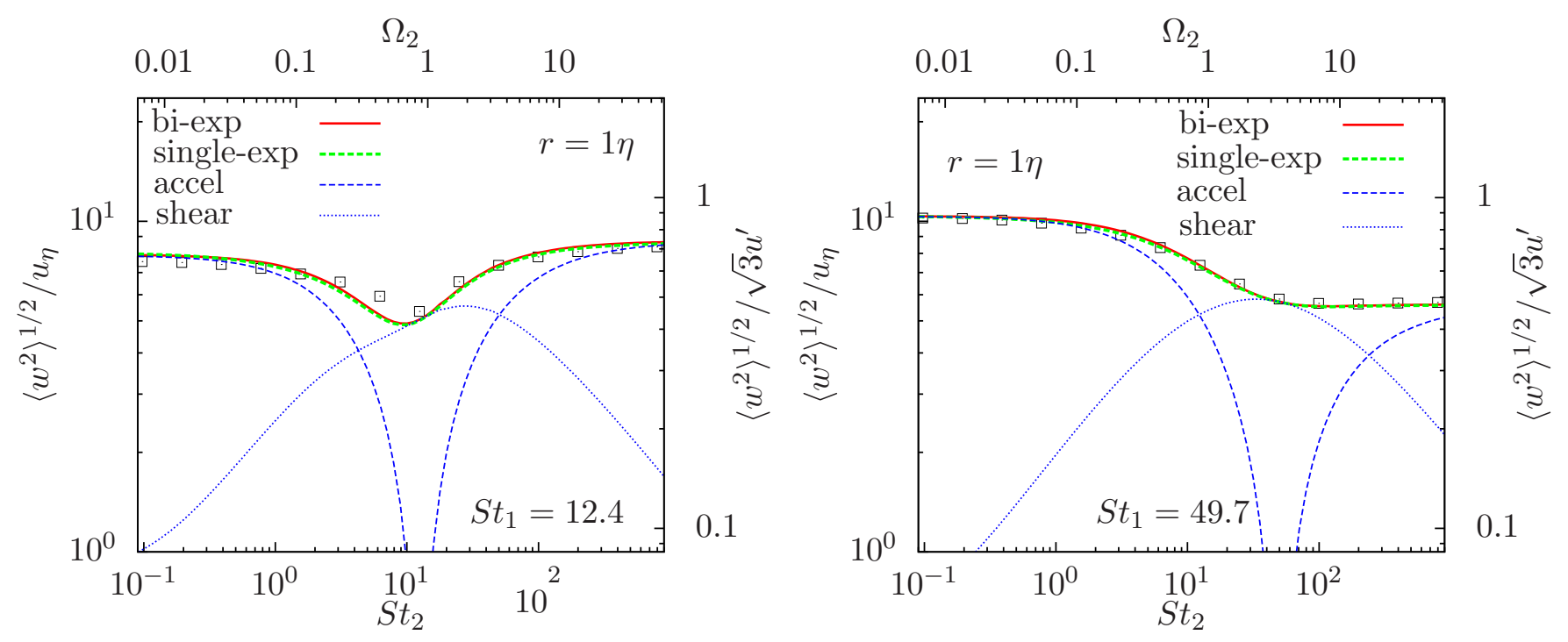

Figure 5. Same as Figure 4, but for $\mathrm{St}_{1}=12.4$ (left panel) and $\mathrm{St}_{1}=49.7$ (right panel). See caption of Figure 4 for details.

(A color version of this figure is available in the online journal.)

with increasing $\mathrm{St}_{2}$. The increase of $R_{\mathrm{p}}$ and/or $T_{\mathrm{p}}$ leads to the increase of the shear term for $\mathrm{St}_{2}$ below $\mathrm{St}_{1}$. On the other hand, as $\mathrm{St}_{2}$ exceeds $\mathrm{St}_{1}$, particle 1 becomes the smaller particle, and at sufficiently large $\mathrm{St}_{2}$, both $R_{\mathrm{p}}$ and $T_{\mathrm{p}}$ become independent of $\mathrm{St}_{2}$ and approach constants. After that, Equation (25) indicates that the shear contribution, $\boldsymbol{w}_{\mathrm{s}}$, would finally decrease as $\mathrm{St}_{2}^{-1 / 2}$, as seen in Figure 4. This decrease corresponds to the reduction in the range of the memory of particle 2 that can contribute to $\mathcal{S}_{i i}$ by the $\Phi_{2}$ term in Equation (27).

In the green dashed line, we used a single-exponential form for both $\Phi_{1}$ and $\Phi_{2}$. The green dashed line is higher than the red solid line and overestimates the data points at small $\mathrm{St}_{2}(\lesssim 0.2)$. We find that the generalized shear contribution is insensitive to the function form of $\Phi_{2}$, and replacing $\Phi_{2}$ with a bi-exponential form does not result in a significant difference in the shear contribution. ${ }^{8}$ The difference between the red and green lines at small $\mathrm{St}_{2}$ is mainly due to the generalized acceleration term. The green line makes use of Equation (19) for the acceleration contribution, which ignores the $z^{2} / 2$ terms in Equation (20) based on the bi-exponential $\Phi_{1}$. In our simulated flow, $z^{2} / 2=0.045$, and we have $\Omega \lesssim 0.07$ for particles with $\mathrm{St} \lesssim 1$. Therefore, for these particles, the $z^{2} / 2$ terms in Equation (20) are not negligible. Physically, for the small particles with $\mathrm{St} \lesssim 1$, the generalized acceleration term has a dependence on the local flow acceleration, $a$, which is not accounted for by the single-exponential temporal correlation. As discussed in Section 3.2.1, for these particles, adopting a bi-exponential $\Phi_{1}$ is needed for an accurate estimate of the acceleration contribution.

We also attempted to test the S-T prediction, $3 a^{2}\left(\tau_{\mathrm{p} 2}-\tau_{\mathrm{p} 1}\right)^{2}+$ $(\bar{\epsilon} / 3 v) r^{2}$, for the 3D variance against our data for small particles with $\mathrm{St}_{1,2} \lesssim 1$. The measured value of the acceleration variance $a^{2}$ in our flow is $4.6 \tau_{\eta}^{-2}$ (see Paper I or Appendix C). We aimed at specifically testing the validity of the acceleration term, $3 a^{2}\left(\tau_{\mathrm{p} 2}-\tau_{\mathrm{p} 1}\right)^{2}$. Thus, at a given $\mathrm{St}_{1}$, we replaced the shear term, $(\bar{\epsilon} / 3 v) r^{2}$, in the S-T formula by the measured value of the monodisperse variance, $\left\langle w^{2}\right\rangle_{\text {mono }}$, at $\mathrm{St}_{2}=\mathrm{St}_{1}$. Comparing

\footnotetext{
8 Similar to the monodisperse case in Paper I, when integrated, the dependence of the double integral in Equation (27) of the generalized shear term on $\Phi_{2}$ condenses to a dependence just on the timescale $T(R)$ in $\Phi_{2}$.
}

$\left(3 a^{2}\left(\tau_{\mathrm{p} 2}-\tau_{\mathrm{p} 1}\right)^{2}+\left\langle w^{2}\right\rangle_{\text {mono }}^{2}\right)^{1 / 2}$ with the data, we find that the acceleration term in the $\mathrm{S}-\mathrm{T}$ prediction works well only for the smallest two particles in our simulation, i.e., for $\mathrm{St}=0.1$ and $\mathrm{St}=0.19$ particles. As mentioned in Section 3.2.1, the generalized acceleration contribution can be approximated by $3 a^{2}\left(\tau_{\mathrm{p} 2}-\tau_{\mathrm{p} 1}\right)^{2}$ only if $\Omega_{1,2} \lesssim 0.08 z^{2}$. With $T_{\mathrm{L}}=14.4$ and $z=0.3$ in our flow, this condition corresponds to $\mathrm{St}_{1,2} \lesssim 0.1$, which is met only by the two smallest particles in our simulation. By including smaller particles, one may further test the S-T prediction for the bidisperse case with $\mathrm{St}_{1,2} \ll 0.1$, where the acceleration term is expected to be determined completely by the flow acceleration, $a$.

Figure 5 shows the simulation results and our model predictions for $\mathrm{St}_{1}=12.4$ (left panel) and $\mathrm{St}_{1}=49.7$ (right panel). The two panels are plotted in the same way as Figure 4 . The assumptions and parameters used in the model predictions are also the same as for the $\mathrm{St}_{1}=0.78$ case in Figure 4 . For $\mathrm{St}_{1}=12.4$ and $\mathrm{St}_{1}=49.7$, there is no significant difference between the predictions with single- or bi-exponential temporal correlation functions. As mentioned in Section 3.2.1, if either of the two particles has $\Omega \gtrsim 3 z^{2}$ (as is the case for $\mathrm{St}_{1}=12.4$ and $\mathrm{St}_{1}=49.7$ ), Equations (19) and (20) for the generalized acceleration term, derived from single- or bi-exponential $\Phi_{1}$, respectively, are close to each other. Together with the fact that the shear contribution is insensitive to the form of $\Phi_{2}$, this explains the coincidence of the red and green lines in both panels of Figure 5.

For $\mathrm{St}_{1}=12.4$, the rms relative velocity changes only slightly with $\mathrm{St}_{2}$. The variation is less than $\simeq 40 \%$. This is of particular interest, as $\mathrm{St}_{1}=12.4$ corresponds to a friction time close to the Lagrangian correlation time, $T_{\mathrm{L}}\left(=14.4 \tau_{\eta}\right)$, of our flow. The small variation of $\left\langle w^{2}\right\rangle$ for $\tau_{\mathrm{p} 1} \simeq T_{\mathrm{L}}$ can be understood by considering three interesting limits: $\mathrm{St}_{2} \rightarrow 0$ (toward the left $y$ axis), $\mathrm{St}_{2}=\mathrm{St}_{1}$, and $\mathrm{St}_{2} \rightarrow \infty$ (the right $y$ axis). In the $\mathrm{St}_{2} \rightarrow 0$ limit, $\left\langle w^{2}\right\rangle^{1 / 2}$ is essentially the particleflow rms relative speed, $w_{\mathrm{f}}^{\prime}$, of particles 1 (see Section 3.2.1). Then, using Equation (6) (or Equation (8)) for $w_{\mathrm{f}}^{\prime}$, we have $\left\langle w^{2}\right\rangle^{1 / 2}=u^{\prime}\left[3 \Omega_{1} /\left(1+\Omega_{1}\right)\right]^{1 / 2} \simeq \sqrt{(3 / 2)} u^{\prime}$ for $\tau_{\mathrm{p} 1} \simeq T_{\mathrm{L}}$ and $\mathrm{St}_{2} \rightarrow 0$. In the opposite limit $\mathrm{St}_{2} \rightarrow \infty$, the velocity of particles 2 is negligible, and its relative velocity with respect to particles 1 is essentially the one-particle velocity, $v^{\prime}$, of particles 1 . Using Equation (4) (or Equation (6)) in Paper I for $v^{\prime}$, we find that 


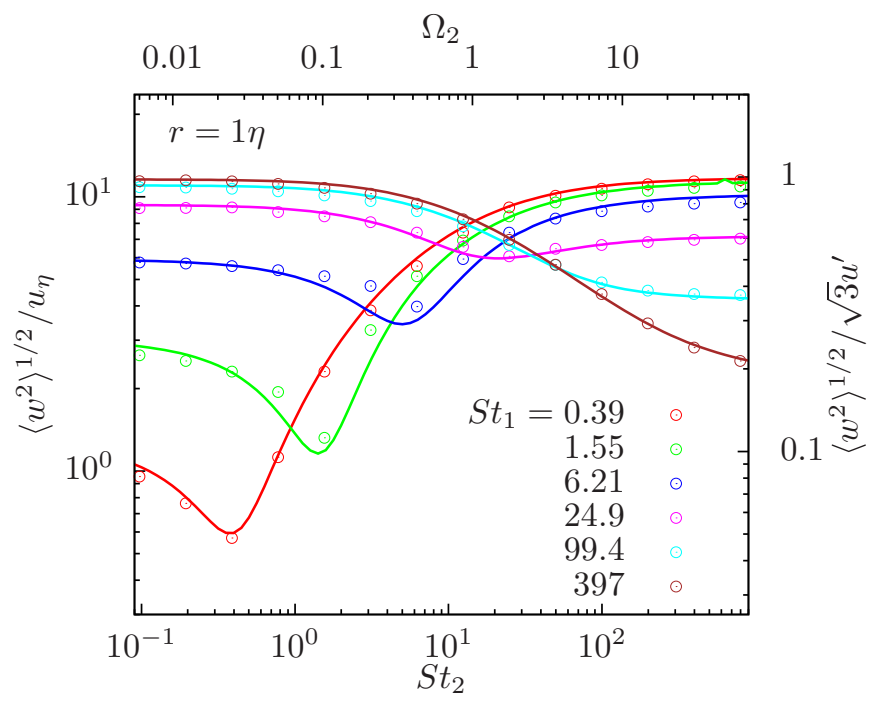

Figure 6. $3 \mathrm{D} \mathrm{rms}$ relative velocity for different values of $\mathrm{St}_{1}$. Data points are simulation results at $r=1 \eta$. Lines are our model predictions using biexponential correlation functions, $\Phi_{1}$ and $\Phi_{2}$.

(A color version of this figure is available in the online journal.)

$\left\langle w^{2}\right\rangle^{1 / 2}=u^{\prime}\left[3 /\left(1+\Omega_{1}\right)\right]^{1 / 2} \simeq \sqrt{(3 / 2)} u^{\prime}$ for $\tau_{\mathrm{p} 1} \simeq T_{\mathrm{L}}$ and $\mathrm{St}_{2} \rightarrow \infty$. This is the same as the estimate for the $\mathrm{St}_{2} \rightarrow 0$ limit. Finally, for the monodisperse case with $\mathrm{St}_{2}=\mathrm{St}_{1}$, we have shown in Paper I that the rms relative speed of identical particles with $\tau_{\mathrm{p}} \simeq T_{\mathrm{L}}$ is about half the flow rms velocity, i.e., $\left\langle w^{2}\right\rangle^{1 / 2} \simeq(\sqrt{3} / 2) u^{\prime}$, which is smaller by a factor of $\sqrt{2}$ than the estimated values for the two extreme limits above. As seen in the left panel of Figure 5, the rms relative speed at the dip $\left(\mathrm{St}_{2}=\mathrm{St}_{1}\right)$ is indeed smaller than at the left and right $y$ axes by $30-40 \%$. The fact that the difference of the three limits is within $\lesssim 40 \%$ explains the small variation of $\left\langle w^{2}\right\rangle^{1 / 2}$ in the whole $\mathrm{St}_{2}$ range. The discussion here is general and not limited to our simulation. We expect that, for $\tau_{\mathrm{p} 1}=T_{\mathrm{L}}$ in any flow, we have $\left\langle w^{2}\right\rangle^{1 / 2} \simeq \sqrt{(3 / 2)} u^{\prime},(\sqrt{3} / 2) u^{\prime}$ and $\sqrt{(3 / 2)} u^{\prime}$ at $\mathrm{St}_{2} \rightarrow 0$, $\mathrm{St}_{2}=\mathrm{St}_{1}$, and $\mathrm{St}_{2} \rightarrow \infty$, respectively.

In the right panel of Figure 5, no dip exists in the data or the model prediction for $\mathrm{St}_{1}=49.7$. Instead, $\left\langle w^{2}\right\rangle^{1 / 2}$ decreases monotonically as $\mathrm{St}_{2}$ increases from 0.1 to 795 . We find that the disappearance of dips actually starts at $\mathrm{St}_{1}=24.9$ (or $\tau_{\mathrm{p} 1}=1.7 T_{\mathrm{L}}$ ). Recall that the dip formation for smaller $\mathrm{St}_{1}$ is due to the tighter velocity correlation between particles of similar size. But if $\tau_{\mathrm{p}}$ is considerably larger than $T_{\mathrm{L}}$, the particle velocities are not significantly correlated even for two particles of exactly the same size (see PP10). Therefore, dips are not expected in the $\left\langle w^{2}\right\rangle^{1 / 2}$ versus $\mathrm{St}_{2}$ curve if $\tau_{\mathrm{p} 1} \gtrsim T_{\mathrm{L}}$.

The fitting quality of our model around the dip for $\mathrm{St}_{1}=12.4$ is not as satisfactory as the $\mathrm{St}_{1}=0.78$ and $\mathrm{St}_{1}=49.7$ cases. The predicted curve overestimates the width of the dip. Furthermore, rather than exactly at $\mathrm{St}_{2}=\mathrm{St}_{1}$, the dip center in the prediction is located at $\mathrm{St}_{2}$ slightly below $\mathrm{St}_{1}$. The same is found for $\mathrm{St}_{1}=3.11$ and $\mathrm{St}_{1}=6.21$. Apparently, this mismatch of the dip center is due to the decrease of the generalized shear term with decreasing $\mathrm{St}_{2}$ at $\mathrm{St}_{2} \leqslant \mathrm{St}_{1}$. The poorer fit of our model around the dips for $\mathrm{St}_{1}=3.11,6.21$, and $\mathrm{St}_{1}=12.4$ than for the $\mathrm{St}_{1} \lesssim 1$ and $\tau_{\mathrm{p} 1} \gtrsim T_{\mathrm{L}}$ cases suggests that the assumptions of the model are the least accurate for inertial-range particles of similar size. The following simplifying assumptions may be responsible for this lower accuracy.
First, as discussed in Appendix A, we ignored a term named $C_{i j}$ in our general formulation for the particle structure function, $S_{\mathrm{p} i j}$, in the bidisperse case. Second, in Section 3.2.1 we neglected the antisymmetric term $B_{i j}^{-}$when evaluating $\mathcal{A}_{i j}$. Both $C_{i j}$ and $B_{i j}^{-}$are related to the asymmetry in the flow velocity statistics along trajectories of the two particles. They are not exactly zero for different particles, and it is possible that they make nonnegligible contributions for inertial-range particles of slightly different sizes. Third, the assumptions in our model for the generalized shear term may be less accurate in the bidisperse case. One example is the assumed separation behavior of particle pairs. As pointed out in Appendix B, how particle pairs of different sizes exactly separate backward in time is largely unknown. A direct study of the pair separation of slightly different particles in the inertial range may help improve the model prediction. Theoretically, an improvement of our model for inertial-range particles of similar size accounting for all the possibilities listed above is of significant interest, as it may further refine the understanding of the physics. On the other hand, from a practical point of view, it may not be particularly useful to develop a high-accuracy model for the rms relative velocity, which, as mentioned earlier, is not directly applicable to the collision statistics (see Paper I). It could be more convenient to simply use the collision statistics measured from the simulation data. Overall, our model is in good agreement with the data despite the small mismatch for inertial-range particles of slightly different sizes, and in general, it provides a successful physical picture for the particle's relative speed in the bidisperse case.

From Figures 4 and 5, we see that the generalized shear term is important only for particles of similar size, and the acceleration term starts to dominate if the Stokes numbers differ by a factor of $\gtrsim 4$. We stress that the interesting limits, $\mathrm{St}_{2} \rightarrow 0, \mathrm{St}_{2}=\mathrm{St}_{1}$, and $\mathrm{St}_{2} \rightarrow \infty$, are very useful delimiters for the behavior of the relative velocity at a given $\mathrm{St}_{1}$.

We summarize the general picture for the bidisperse relative velocity in Figure 6, which shows $\left\langle w^{2}\right\rangle^{1 / 2}$ at $r=1 \eta$ for six values of $\mathrm{St}_{1}$ ranging from 0.39 to 397 . Lines are the model predictions using bi-exponential $\Phi_{1}$ and $\Phi_{2}$ and the same separation behavior as in Figures 4 and 5. There is a clear difference in the behavior of $\left\langle w^{2}\right\rangle^{1 / 2}$ for $\tau_{\mathrm{p} 1} \lesssim T_{\mathrm{L}}$ and $\tau_{\mathrm{p} 1} \gtrsim T_{\mathrm{L}}$. For $\tau_{\mathrm{p} 1} \lesssim T_{\mathrm{L}}$, a dip exists at $\mathrm{St}_{2}=\mathrm{St}_{1}$, and away from the dip, $\left\langle w^{2}\right\rangle^{1 / 2}$ increases toward $\mathrm{St}_{2} \rightarrow 0$ and $\mathrm{St}_{2} \rightarrow \infty$. On the other hand, for $\tau_{\mathrm{p} 1} \gtrsim T_{\mathrm{L}}$, there are no dips, and the relative speed decreases monotonically with increasing $\mathrm{St}_{2}$. In the $\mathrm{St}_{2} \rightarrow 0$ limit, i.e., on the left $y$ axis, $\left\langle w^{2}\right\rangle^{1 / 2}$ corresponds to the particle-flow relative velocity for particles 1 and increases with increasing $\mathrm{St}_{1}$ (see Equation (8) and Figure 3). In the other limit $\mathrm{St}_{2} \rightarrow \infty$ (the right $y$ axis), the relative velocity decreases monotonically with increasing $\mathrm{St}_{1}$, corresponding to the decrease of the one-particle relative velocity of particles 1 with increasing $\tau_{\mathrm{p} 1}$ (see Equation (6) and Figure 5 of Paper I). For $\tau_{\mathrm{p} 2} \simeq T_{\mathrm{L}}$ (i.e., $\mathrm{St}_{2} \simeq 14.4$ ), the change of $\left\langle w^{2}\right\rangle^{1 / 2}$ with $\mathrm{St}_{1}$ in the vertical direction is slight, corresponding to the small variation of $\left\langle w^{2}\right\rangle^{1 / 2}$ as a function of $\mathrm{St}_{2}$ in the horizontal direction for $\mathrm{St}_{1}=12.4$ (see the left panel of Figure 5).

\subsection{The rms Relative Velocity at Fixed Stokes Ratios}

Instead of fixing one of the Stokes numbers, it may also be convenient to analyze the relative velocity of particle pairs with a fixed Stokes number ratio, $f$. As a reminder, $f$ is defined as the ratio of the lower Stokes number, $\mathrm{St}_{\ell}\left(\equiv \min \left(\mathrm{St}_{1}, \mathrm{St}_{2}\right)\right)$, to 
the higher one, $\mathrm{St}_{h}\left(\equiv \max \left(\mathrm{St}_{1}, \mathrm{St}_{2}\right)\right)$, so that $0 \leqslant f \leqslant 1$. In Figure 7, we show the 3D rms relative speed at $r=1 \eta$ as a function of $\mathrm{St}_{h}$ for different values of $f$. The monodisperse case (black diamonds) corresponds to $f=1$. As discussed in Paper I, for small identical particles at St $\lesssim 1$, the relative velocity at $r=1 \eta$ is mainly determined by the local flow velocity difference and is essentially independent of St. As St increases above 1 , the particle memory of the spatial flow velocity difference in the past becomes important, and the relative velocity starts to increase. $\mathrm{A} \mathrm{St}^{1 / 2}$ scaling (dotted line segment) was predicted for inertial-range particles by various models. For large particles with $\tau_{\mathrm{p}} \gg T_{\mathrm{L}}$, the memory/correlation time of the flow at the largest scales is shorter than the particle memory, and the flow memory cutoff (by the $\Phi_{2}$ term) causes a decrease of the relative velocity with $\mathrm{St}$ as $\mathrm{St}^{-1 / 2}$.

The black squares show the particle-flow relative velocity, $\left\langle w_{\mathrm{f}}^{2}\right\rangle^{1 / 2}$, corresponding to $f \rightarrow 0$. For a consistent comparison, $\left\langle w_{\mathrm{f}}^{2}\right\rangle^{1 / 2}$ is measured here at the same distance $(r=1 \eta)$ as in the particle-particle cases. ${ }^{9}$ We used the TSC interpolation to obtain the flow velocities at a separation $r$ from the position of each particle in the three orthogonal directions of the simulation grid. The black dotted line shows our model prediction. To compute the prediction, we set one of the friction times in Equation (27) to zero, which reduces the equation to a single integral. We used exactly the same assumptions and parameters as in the predictions for the particle-particle relative velocity in the bidisperse case. The prediction is in good agreement with the data points.

The color data points show the simulation results for $(1 / 16) \leqslant$ $f \leqslant(1 / 2)$, which all lie in between the particle-flow relative velocity $(f=0)$ and the monodisperse case $(f=1)$. Due to the contribution of the generalized acceleration term, the relative velocity increases as $f$ decreases. At a given $\mathrm{St}_{h}$, the increase of $\left\langle w^{2}\right\rangle^{1 / 2}$ with decreasing $f$ corresponds to the increase of the data points or lines in Figure 6 toward the left $y$ axis, i.e., at $\mathrm{St}_{2} \leqslant \mathrm{St}_{1}$. The color lines in the figure are our model predictions using bi-exponential temporal correlation functions and a two-phase separation behavior with a Richardson constant of $g=1.6$ (i.e., the same as the lines in Figures 4, 5, and 6). The model prediction matches the data reasonably well. $\mathrm{As} \mathrm{St}_{h} \rightarrow 0$, all the data points and lines appear to converge to the same value. At any given $f$, both particles become tracer particles as $\mathrm{St}_{h} \rightarrow 0$. Therefore, in the $\mathrm{St}_{h} \rightarrow 0$ limit, the relative velocity always approaches the spatial flow velocity difference across the particle distance, $r$ (see the shear term in the S-T formula).

Like the monodisperse case, $\left\langle w^{2}\right\rangle^{1 / 2}$ first increases with $\mathrm{St}_{h}$, reaches a maximum, and finally decreases. We find that in general the peak of $\left\langle w^{2}\right\rangle^{1 / 2}$ lies in the range $T_{\mathrm{L}} \lesssim \tau_{\mathrm{p}, \mathrm{h}} \lesssim T_{\mathrm{L}} / f$ (or equivalently $f T_{\mathrm{L}} \lesssim \tau_{\mathrm{p}, 1} \lesssim T_{\mathrm{L}}$ ). In this range, the variation of $\left\langle w^{2}\right\rangle^{1 / 2}$ is small. The value of $\mathrm{St}_{h}$ at which $\left\langle w^{2}\right\rangle^{1 / 2}$ peaks increases with decreasing $f$. For $\mathrm{St}_{\ell} \gg T_{\mathrm{L}}\left(\mathrm{St}_{h} \gg T_{\mathrm{L}} / f\right)$, $\left\langle w^{2}\right\rangle^{1 / 2}$ is expected to decrease as $\mathrm{St}_{h}^{-1 / 2}$ (see Equation (13) and the discussion below).

The two dotted line segments denote a $\mathrm{St}_{h}^{1 / 2}$ scaling for $\mathrm{St}_{h}$ in the inertial range. Such a scaling has been predicted for inertialrange particles of equal size $(f=1)$ by various models (see Paper I). The same scaling was also predicted by our model for

\footnotetext{
9 Note that the particle-flow relative velocity, $w_{\mathrm{f}}^{\prime}$, shown in Figure 3, is at zero distance. The data points for $w_{\mathrm{f}}^{\prime}$ at $r=0$ and $\left\langle w_{\mathrm{f}}^{2}\right\rangle^{1 / 2}$ at $r=1 \eta$ are found to coincide at St $\gtrsim 1$. But at St $\lesssim 1,\left\langle w_{\mathrm{f}}^{2}\right\rangle^{1 / 2}$ at $r \simeq 1 \eta$ is slightly larger due to the contribution of the shear term. The shear contribution to $\left\langle w_{\mathrm{f}}^{2}\right\rangle$ for the $\mathrm{St} \lesssim 1$ particles is approximately $(\bar{\epsilon} / 3 v) r^{2}$.
}

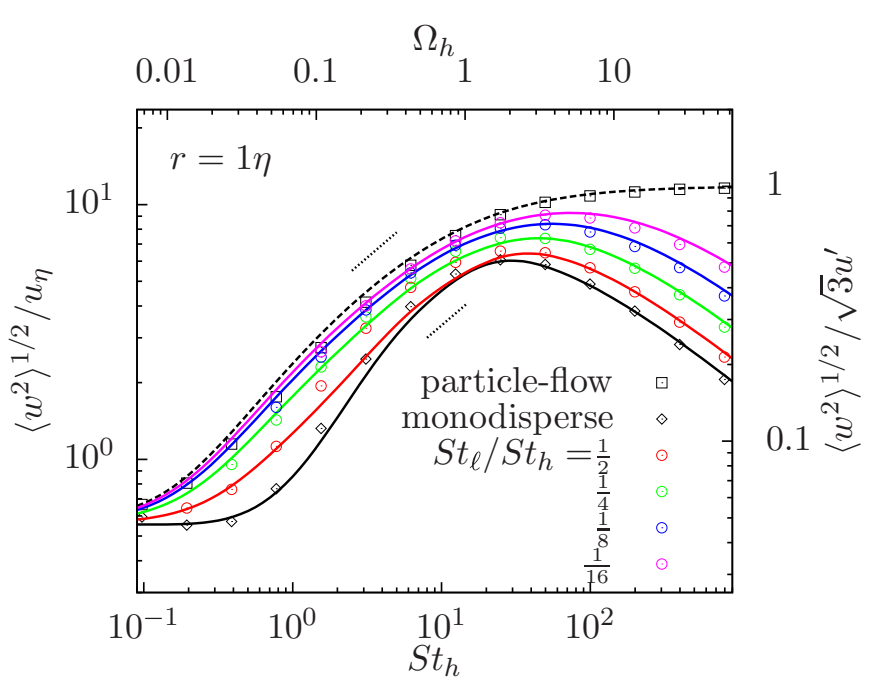

Figure 7. 3D rms relative velocity at $r=1 \eta$ for particle pairs with fixed Stokes ratios, $f \equiv \mathrm{St}_{\ell} / \mathrm{St}_{h}=(1 / 2)$ (red), $1 / 4$ (green), 1/8 (blue), and 1/16 (magenta). Black diamonds and squares correspond to the monodisperse case $(f=1)$ and the particle-flow relative velocity $(f=0)$. The lines show our model predictions. The two black dotted line segments correspond to a $\mathrm{St}_{h}^{1 / 2}$ scaling.

(A color version of this figure is available in the online journal.)

the relative velocity, $w_{f}$, between the flow and inertial-range particles (i.e., for $f=0$; see Section 2 and 5). This suggests the possibility of a universal $\mathrm{St}_{h}^{1 / 2}$ scaling in the inertial range for any $f$ between 0 and 1 . Using Equation (21) and the assumption $\Delta \boldsymbol{u}_{\mathrm{T}} \simeq \Delta \boldsymbol{u}_{\mathrm{L}}$, we see that, for a fixed $f$, the acceleration term, $\boldsymbol{w}_{\mathrm{a}}$, would scale as $\mathrm{St}_{h}^{1 / 2}$ for $\tau_{\mathrm{p}, \mathrm{h}}$ in the inertial range. This could also be shown from Equation (20): at a given $f$, the acceleration contribution, $A$, to the relative velocity variance goes linearly with $\Omega_{h}$ if $z^{2} / 2 \ll \Omega_{h} \ll 1$. Therefore, the $\mathrm{St}_{h}^{1 / 2}$ scaling is expected for $f \lesssim(1 / 4)$, where the acceleration effect dominates. Considering the high probability that the shear term, $\boldsymbol{w}_{\mathrm{s}}$, in the monodisperse case scales as $\mathrm{St}^{1 / 2}$ in the inertial range, it may be generally true that the scaling applies to any $f$. However, to observe a convincing $\mathrm{St}_{h}^{1 / 2}$ scaling, the flow must have an extended inertial range. Higher-resolution simulations are needed to verify if a $\mathrm{St}_{h}^{1 / 2}$ scaling holds in general for any values of $f$ in the range $0 \leqslant f \leqslant 1$.

In Figure 8 , we show the rms relative velocity for $f=(1 / 2)$ (left panel) and $f=(1 / 8)$ (right panel) in more detail. The data points and solid lines are the same as the corresponding ones in Figure 7. The discrepancy between the data and our model prediction is largest for $f=(1 / 2)$. In this case, our model underestimates the data points by $\simeq 20 \%$ for $\mathrm{St}_{h}=3.11$ and 6.21 and by $\simeq 15 \%$ for $\mathrm{St}_{2}=1.55$ and 12.4. On the other hand, the model prediction agrees with the data quite well for smaller $\left(\mathrm{St}_{h} \lesssim 1\right)$ or larger $\left(\tau_{\mathrm{p}, \mathrm{h}} \gtrsim T_{\mathrm{L}}\right)$ particles. A detailed discussion on the possible reasons for the discrepancy for inertial-range particles of similar size was given in Section 6.1. The agreement improves with decreasing $f$, and at $f=(1 / 4)$ the discrepancy is $<10 \%$.

The dashed and dotted lines in Figure 8 plot the generalized shear and acceleration contributions in our model predictions. At $f=(1 / 2)$, the relative velocity is mainly contributed by the shear term, except for $0.4 \leqslant \mathrm{St}_{h} \leqslant 3$. The contribution of the acceleration term increases with decreasing $f$, and at $f \leqslant(1 / 8)$, the rms relative velocity is dominated by the acceleration contribution. 
$0.01 \quad 0.1 \quad \Omega_{h} \quad 10$

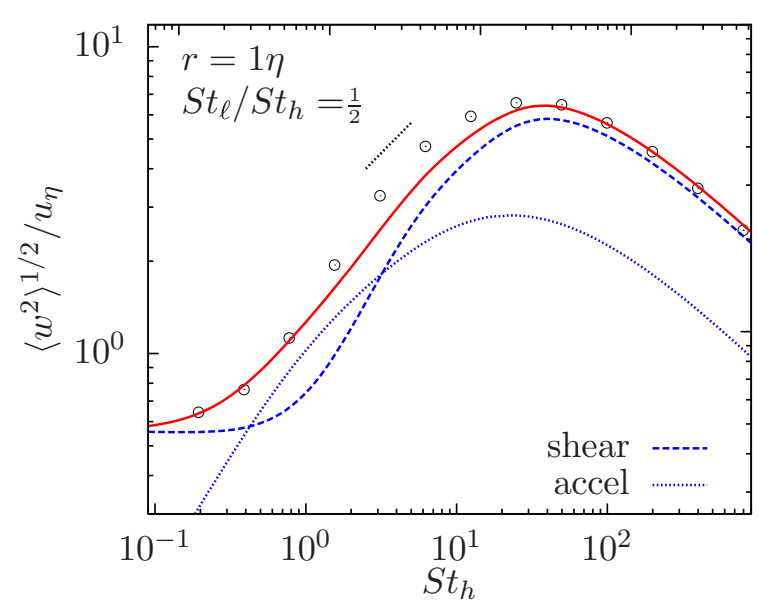

1

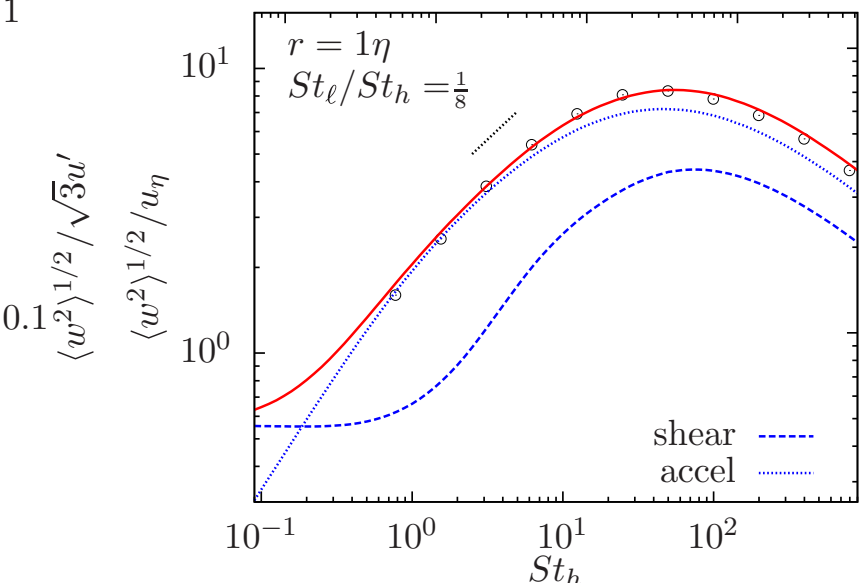

1

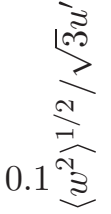

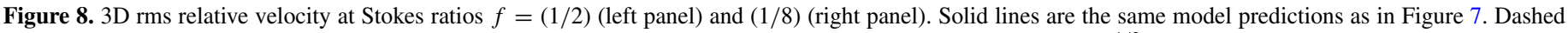
and dotted lines are the generalized shear and acceleration contributions, respectively. Dotted line segments denote $\mathrm{St}_{h}^{1 / 2} \mathrm{scaling}$.

(A color version of this figure is available in the online journal.)

The behaviors of the generalized acceleration and shear contributions as a function of $\mathrm{St}_{h}$ can be understood from Equations (21) and (25) for $\boldsymbol{w}_{\mathrm{a}}$ and $\boldsymbol{w}_{\mathrm{s}}$, respectively. Equation (21) indicates that, at a fixed $f, w_{\mathrm{a}}$ increases with $\mathrm{St}_{h}$ for $\tau_{\mathrm{p}, \mathrm{h}} \lesssim T_{\mathrm{L}}$ because $\Delta_{\mathrm{T}} \boldsymbol{u}\left(\tau_{\mathrm{p}, \mathrm{h}}\right)$ increases with $\tau_{\mathrm{p}, \mathrm{h}}$. Due to the $\left(1+\Omega_{\ell}\right)^{-1 / 2}$ (or $\left(1+f \Omega_{h}\right)^{-1 / 2}$ ) factor in Equation (21), $\boldsymbol{w}_{\mathrm{a}}$ decreases as $\mathrm{St}_{h}^{-1 / 2}$ at $\tau_{\mathrm{p}, \mathrm{h}} \gg T_{\mathrm{L}} / f$. These are indeed observed in the dotted lines in Figure 8 . The acceleration term is roughly constant for $T_{\mathrm{L}} \lesssim \tau_{\mathrm{p}, \mathrm{h}} \lesssim T_{\mathrm{L}} / f$ because in this range the amplitude of $\Delta_{\mathrm{T}} \boldsymbol{u}\left(\tau_{\mathrm{p}, \mathrm{h}}\right)$ is $\simeq u^{\prime}$ and $\Omega_{\ell} \lesssim 1$. In order to understand the shear contribution, we calculate the rms of $\boldsymbol{w}_{\mathrm{s}}$ from Equation (25), using the method described in Footnote 4 to estimate the primary distance, $R_{\mathrm{p}}$, and the timescale, $T_{\mathrm{p}}$. The calculation shows that the rms of $\boldsymbol{w}_{\mathrm{s}}$ is consistent with $\sqrt{\mathcal{S}_{i i}}$ computed from the double-integral Equation (27), supporting the validity of Equation (25) as an approximate estimate for the shear term. At small $\mathrm{St}_{h}$, both $R_{\mathrm{p}}$ and $T_{\mathrm{p}}$ in Equation (25) increase with $\mathrm{St}_{h}$, and thus the shear contribution increases. $\mathrm{As} \mathrm{St}_{h}$ keeps increasing, $T_{\mathrm{p}}$ finally reaches the maximum value $\left(T_{\mathrm{L}}\right)$ when the friction time, $\tau_{\mathrm{p}, 1}$, of the smaller particle increases up to $\simeq T_{\mathrm{L}}$. At $\tau_{\mathrm{p}, 1} \gtrsim T_{\mathrm{L}}, T_{\mathrm{p}}$ stays constant $\left(=T_{\mathrm{L}}\right)$, and the $\left[T_{\mathrm{p}} /\left(T_{\mathrm{p}}+\tau_{\mathrm{p}, \mathrm{h}}\right)\right]^{1 / 2}$ term in Equation (25) causes $\boldsymbol{w}_{\mathrm{s}}$ to decrease with $\mathrm{St}_{h}$. The condition $\tau_{\mathrm{p}, 1} \gtrsim T_{\mathrm{L}}$ corresponds to $\Omega_{\ell} \gtrsim 1$ or $\Omega_{h} \gtrsim 1 / f$. This explains why the peak of the shear contribution occurs at larger $\mathrm{St}_{h}$ for smaller $f$. Similar to the acceleration term, the shear contribution, $\sqrt{S_{i i}}$, decreases as $\propto \mathrm{St}_{h}^{-1 / 2}$ at $\Omega_{h} \gg 1 / f$.

\subsection{Dependence on the Particle Distance}

In Figure 9, we show the 3D rms relative velocity at different particle distances. The left and right panels plot $\left\langle w^{2}\right\rangle^{1 / 2}$ at fixed values of $\mathrm{St}_{1}$ and fixed Stokes ratios $f$, respectively. The solid, dashed, and dotted lines are our model predictions for $r=1$, $1 / 2$, and $1 / 4 \eta$ using bi-exponential temporal correlations and a two-phase behavior for the particle separation backward in time. In the Richardson phase, $g$ is set to $1.6,1.3$, and 1.0 for $r=1$, $1 / 2$, and $1 / 4 \eta$, respectively. These values of $g$ are the same as those used in Paper I that best fit the rms relative velocity of equal-size particles at the corresponding distances. As discussed in Paper I, our choice of smaller Richardson constant $g$ at smaller $r$ is based on the observation that the directly measured value of $g$ for the backward-in-time pair separation of tracer particles in our simulated flow decreases with decreasing initial distance $r$. This is likely due to the limited inertial range of the simulated flow, and $g$ may reach a universal constant value if the flow's Reynolds number is sufficiently larger (Paper I). It would be convenient if one can set $g$ to a fixed constant in our model. However, the measured $g$ for tracer particles suggests otherwise, and allowing $g$ to change with $r$ for inertial particles in our flow appears to be a more natural choice, as fixing $g$ to a single value may leave the misleading impression that $g$ is strictly constant with $r$ in our simulated flow. We thus pursued best fits of our model prediction to the simulation data by varying $g$ with $r$. The fact that the best-fit $g$ decreases with $r$ is consistent with the direct measurement of $g$ for tracer particles. In protoplanetary turbulence, the Reynolds number is much larger than in our simulation, and one may expect a constant $g$ at different values of $r$. However, this expectation and the exact value of $g$ in flows with much larger Re needs to be examined by numerical simulations at considerably higher resolution.

In the left panel of Figure 9, we see that, for $\mathrm{St}_{1}<6.2$, the dips at $\mathrm{St}_{2}=\mathrm{St}_{1}$ become deeper with decreasing $r$. This corresponds to the $r$ dependence of the relative velocity in the monodisperse case (see the $f=1$ case in the right panel). For small equal-size particles in the S-T limit, the relative velocity decreases with decreasing $r$, as it is determined largely by the local flow velocity difference across $r$. As St increases, the particle memory of the spatial flow velocity difference in the past provides larger contribution, and the $r$ dependence becomes weaker. At $S t \gtrsim 6.2$, the particle memory time is significant, and the particle separation a friction time ago is insensitive to its initial value, $r$. This explains the $r$ independence of the dip for $\mathrm{St}_{1}=6.2$ in the left panel, as well as the $r$ independence of the $f=1$ case at $\mathrm{St} \gtrsim 6.2$ in the right panel.

In the bidisperse case, the generalized acceleration term is independent of the particle distance (see Section 3.2 and Appendix A), and its presence reduces the $r$ dependence of $\left\langle w^{2}\right\rangle^{1 / 2}$. In the left panel of Figure 9, the relative speed is less dependent on $r$, as $\mathrm{St}_{2}$ moves away from the dip center. Clearly, toward both sides of the dips, the contribution from 

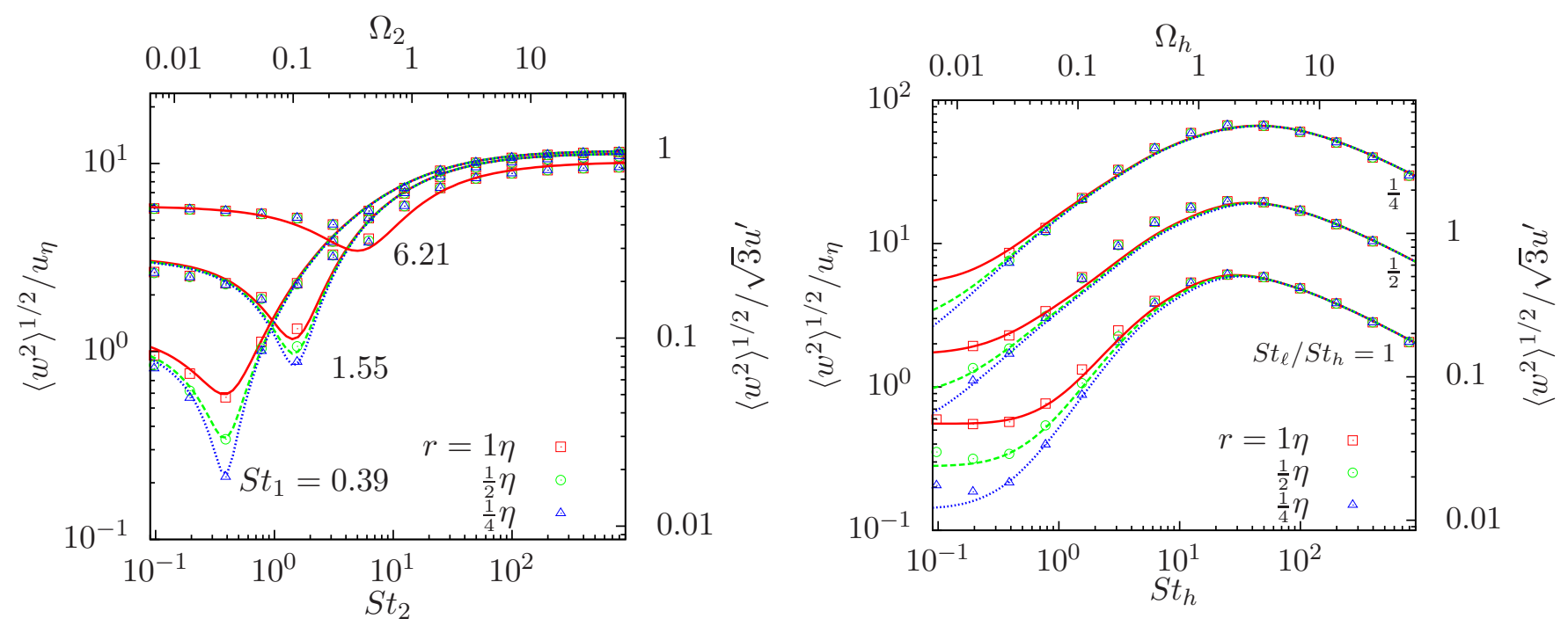

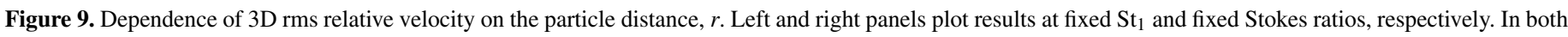

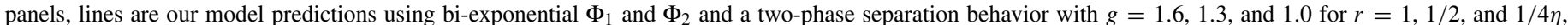
respectively. For clarity, the data points and lines in the right panel for $f=(1 / 2)$ and $(1 / 4)$ are shifted upward by a factor of three and nine, respectively.

(A color version of this figure is available in the online journal.)

the generalized acceleration term increases. For $\mathrm{St}_{1} \gtrsim 6.2$, the generalized shear term is already independent of $r$ for $\mathrm{St}_{2}$ around $\mathrm{St}_{1}$, and this suggests that, if one of the Stokes numbers is larger than $6.2,\left\langle w^{2}\right\rangle^{1 / 2}$ is $r$-independent.

The same behavior is seen in the right panel: the $r$ dependence of $\left\langle w^{2}\right\rangle^{1 / 2}$ becomes weaker as the Stokes ratio $f$ decreases. The weaker $r$ dependence in the bidisperse case makes the evaluation of the collision statistics much easier than in the monodisperse case. As discussed in the Introduction (see also Paper I), dust particles in protoplanetary disks are nearly point-like, and in principle one needs to extrapolate the measured statistics to the $r \rightarrow 0$ limit before applying it to dust particle collisions. In Paper I, we found that, for equal-size particles with $\mathrm{St} \lesssim 1$, the extrapolation is very challenging because their relative velocity has a significant $r$ dependence that may persist to very small $r$. In the bidisperse case, the relative velocity of small particles would converge once the $r$-independent acceleration contribution dominates, making it considerably easier to directly compute the collision statistics from the numerical simulations.

We point out that, in principle, the Richardson constant $g$ is not a free parameter, as it is physically controlled by the properties of the turbulent flow and the particle dynamics. However, the exact value of $g$ for inertial particles is largely unknown, and its behavior with decreasing $r$ and the flow Reynolds number, Re, is currently an open question. We thus treated $g$ essentially as a free parameter in our model prediction. Our assumption concerning particle separation will be refined and improved with the help of future numerical studies that directly investigate the backwardin-time separation of inertial particles. Due to the uncertainty of $g$, it is useful to examine the dependence of our model prediction on $g$. The $g$ dependence of our model for equal-size particles was shown in the left panel of Figure 2 in PP10. It was found in PP10 that the dependence on $g$ is rather weak, and for inertial-range particles, the model prediction for $\left\langle w^{2}\right\rangle^{1 / 2}$ scales with $g$ as $g^{1 / 3}$. Note that the right panel of Figure 2 in PP10 assumed a different separation behavior and a different Reynolds number than in the current work. Repeating the same analysis of $g$ dependence with the separation behavior adopted in this work and Re in our simulated flow, we found a similar weak dependence on $g$ : the predicted $\left\langle w^{2}\right\rangle^{1 / 2}$ decreases only by $\lesssim 20 \%$ for $0.8 \lesssim \mathrm{St} \lesssim 10$, as $g$ decreases by each factor of two, consistent with the $g^{1 / 3}$ dependence. This weak $g$ dependence, to some degree, alleviates the problem of uncertainties in $g$ and its behavior with $r$ and Re. In order to estimate the effects of the uncertainties in $g$, we also attempted to compare our model with $g$ fixed at a single value of 1.6 against simulation results for all $r$. It turns out that, due to the weak $g$ dependence, our model with $g=1.6$ is in acceptable agreement with the data for all $r \gtrsim(1 / 4) \eta$. For example, for equal-size particles, changing $g$ from the best value of 1.0 for $r=(1 / 4) \eta$ to 1.6 only leads to a $\lesssim 15 \%$ overestimate for the St range $0.4 \lesssim S t \lesssim 3$. Also $g$ does not affect the acceleration term, and thus the $g$ dependence is even weaker for particles of different sizes. If the Stokes numbers differ by a factor of $\gtrsim 2$, our model prediction is barely affected as $g$ is changes from 1 (or 1.3) to 1.6. We emphasize that fixing $g$ to a single value here is for an illustration, as there is no evidence that $g$ is constant in our flow. As mentioned earlier, $g$ may be constant with $r$ in realistic flows with much larger Re.

\subsection{The Radial and Tangential rms Relative Speeds}

In Figure 10, we show the rms relative speeds in the radial $\left(\left\langle w_{\mathrm{r}}^{2}\right\rangle^{1 / 2}\right.$; filled symbols $)$ and tangential $\left(\left\langle w_{\mathrm{t}}^{2}\right\rangle^{1 / 2}\right.$; open symbols $)$ directions at $r=1,1 / 2$ and $1 / 4 \eta$. In the left panel, we fix $\mathrm{St}_{1}$ at $0.19,0.78$, and 3.21, while the right panel shows fixed Stokes ratios, $f=1,1 / 2$, and $1 / 4$. Results on the radial and tangential relative speeds in the monodisperse case (the $f=1$ case in the right panel of Figure 10) were already discussed in Paper I. The S-T formula predicts that the tangential rms is larger than the radial one by $\sqrt{2}$ for identical particles with St $\ll 1$ (see Equation (12)). This factor originates from the difference in the longitudinal and tangential structure functions of incompressible turbulence. However, in Paper I we found that, at $1 / 4 \eta \leqslant r \leqslant 1 \eta$, the tangential-to-radial rms ratio is about 1.1 for the smallest particles $(\mathrm{St}=0.1)$ in our simulation, and it decreases to unity at $\mathrm{St} \simeq 1$, above which $\left\langle w_{\mathrm{t}}^{2}\right\rangle^{1 / 2}$ and $\left\langle w_{\mathrm{r}}^{2}\right\rangle^{1 / 2}$ are equal (see the $f=1$ data in the right panel). One reason for the near equality of $\left\langle w_{\mathrm{t}}^{2}\right\rangle^{1 / 2}$ and $\left\langle w_{\mathrm{r}}^{2}\right\rangle^{1 / 2}$ for the St $\lesssim 1$ particles is 

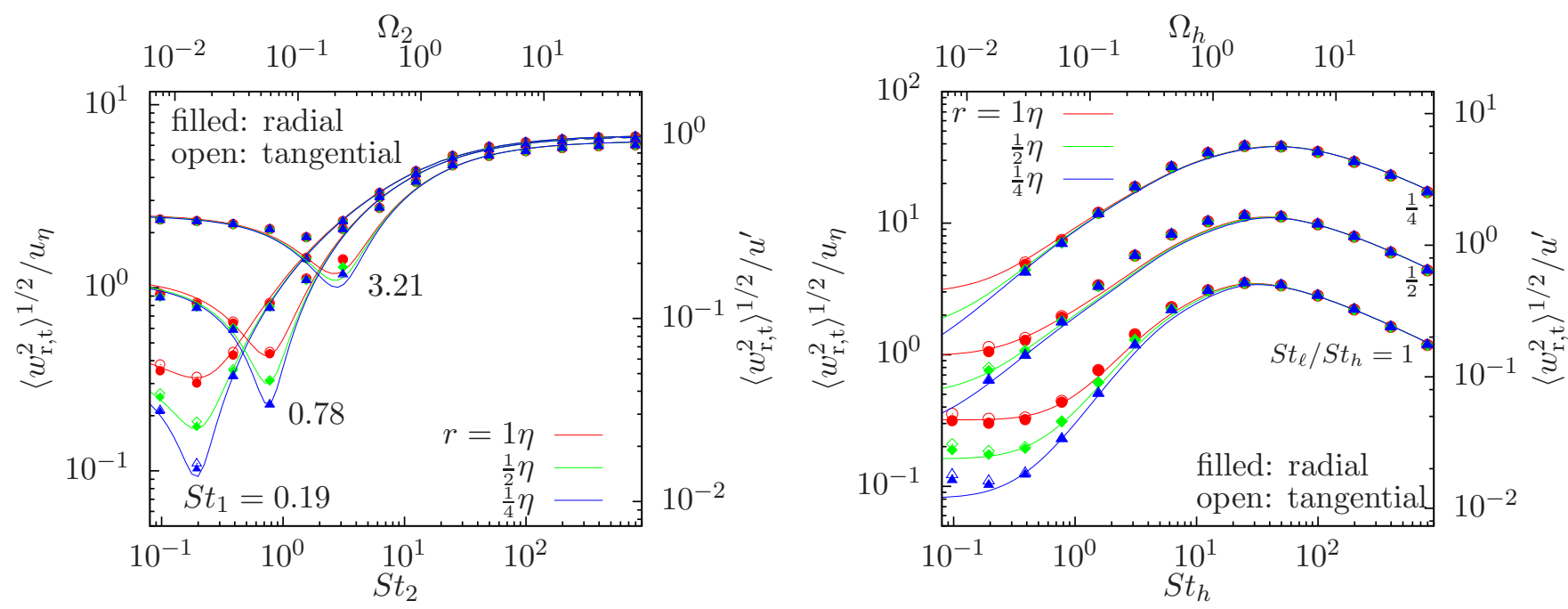

Figure 10. Radial (filled symbols) and tangential (open symbols) rms relative speeds at different $r$. Left and right panels show results at fixed $\mathrm{St}_{1}$ and fixed Stokes ratios, respectively. The lines are our model predictions using the same parameters as in Figure 9. Equation (24) is adopted for $\left\langle S_{i j}\right\rangle_{\text {ang }}$, which predicts $\left\langle w_{\mathrm{r}}^{2}\right\rangle=\left\langle w_{\mathrm{t}}^{2}\right\rangle=(1 / 3)\left\langle w^{2}\right\rangle$. For clarity, the data points and lines in the right panel for $f=(1 / 2)$ and $(1 / 4)$ are shifted upward by a factor of three and nine, respectively. (A color version of this figure is available in the online journal.)

the deviation of their trajectories from the flow elements, which randomizes the direction of the relative velocity with respect to the particle separation, $\boldsymbol{r}$. For larger particles, the effect of the particle memory of the spatial flow velocity difference in the past and the stochastic particle separation backward in time also tends to equalize $\left\langle w_{\mathrm{r}}^{2}\right\rangle^{1 / 2}$ and $\left\langle w_{\mathrm{t}}^{2}\right\rangle^{1 / 2}$.

In the bidisperse case, the acceleration contributions to the radial and tangential components are equal both in the S-T formula (Equation (12)) and in our model prediction (see Equation (17)). Therefore, the tangential-to-radial ratio for different particles is expected to be closer to unity than the monodisperse case. In the left panel of Figure 10, we see that $\left\langle w_{\mathrm{t}}^{2}\right\rangle^{1 / 2}$ is slightly larger than $\left\langle w_{\mathrm{r}}^{2}\right\rangle^{1 / 2}$ at the dip center for $\mathrm{St}_{1}=0.19$. As $\mathrm{St}_{2}$ moves away from $\mathrm{St}_{1}=0.19$, the acceleration contribution increases, leading to a decrease in the difference between $\left\langle w_{\mathrm{r}}^{2}\right\rangle^{1 / 2}$ and $\left\langle w_{\mathrm{t}}^{2}\right\rangle^{1 / 2}$. There is also a general trend for $\left\langle w_{\mathrm{r}}^{2}\right\rangle^{1 / 2}$ and $\left\langle w_{\mathrm{t}}^{2}\right\rangle$ to equalize as $r$ decreases. For the bidisperse case, this corresponds to a relative increase in the acceleration contribution, as the shear term decreases with decreasing $r$ for small particles in the S-T limit. The same behavior is seen in the right panel. If one Stokes number is larger than $\simeq 1$, the tangential-to-radial ratio is unity, since $\left\langle w_{\mathrm{r}}^{2}\right\rangle^{1 / 2}$ and $\left\langle w_{\mathrm{t}}^{2}\right\rangle^{1 / 2}$ are already equal in the monodisperse case with $\mathrm{St} \gtrsim 1$.

Due to the near equality of the radial and tangential rms speeds for all Stokes pairs in our simulation, we adopt Equation (24) for the angular average of the trajectory structure tensor, $\left\langle S_{\mathrm{T} i j}\right\rangle_{\mathrm{ang}}$, which predicts $\left\langle w_{\mathrm{r}}^{2}\right\rangle=\left\langle w_{\mathrm{t}}^{2}\right\rangle=(1 / 3)\left\langle w^{2}\right\rangle$. The lines in Figure 10 are our model predictions from this equation, ${ }^{10}$ which are in good agreement with the data points. Again, we adopted bi-exponential $\Phi_{1}$ and $\Phi_{2}$ and a two-phase separation behavior with $g=1.6,1.3$ and 1.0 for $r=1,1 / 2$, and $1 / 4 \eta$, respectively. The $r$ dependence of $\left\langle w_{\mathrm{r}}^{2}\right\rangle^{1 / 2}$ and $\left\langle w_{\mathrm{t}}^{2}\right\rangle^{1 / 2}$ is similar to that of the 3D rms shown in Figure 9.

To summarize Sections 6.3 and 6.4, we found that the interesting features of the rms relative velocity for equal-size particles discussed in Paper I become weaker in the bidisperse

\footnotetext{
10 As discussed in Appendix B, using Equation (26) in Paper I for $\left\langle S_{i j}\right\rangle_{\text {ang }}$ would recover the $\mathrm{S}-\mathrm{T}$ prediction for small particles, which, however, gives poorer fits to the data.
}

case. The generalized acceleration term is rather featureless: it is independent of the particle distance and provides equal contributions to the radial and tangential components of the relative velocity. As the acceleration contribution increases with increasing Stokes number difference, both the $r$ dependence and the tangential-to-radial ratio decrease. In Appendix D, we follow Paper I to split particle pairs at given distances into two groups with negative and positive radial relative speed, corresponding to particles approaching and separating from each other, respectively. This division is of interest because only approaching particle pairs may lead to collisions. Paper I found that, for equal-size particles with St $\lesssim 6$, approaching pairs have larger relative speed than separating ones. In the bidisperse case, the acceleration contribution is independent of the relative motions of the two particles as it depends only on the flow velocity statistics along individual particle trajectories. Therefore, as shown in Appendix D, the asymmetry between approaching and separating pairs is weaker in the bidisperse case than in the monodisperse one.

\section{SUMMARY AND CONCLUSIONS}

We have investigated the relative velocity of inertial particles suspended in turbulent flows, extending our earlier work on equal-size particles (Pan \& Padoan 2013; Paper I) to the general bidisperse case for different particles of arbitrary size. We have made use of the same numerical simulation presented in Paper I, which evolved 14 species of inertial particles in a simulated turbulent flow. The particle friction time, $\tau_{\mathrm{p}}$, ranges from $0.1 \tau_{\eta}(\mathrm{St}=0.1)$ to $54 T_{\mathrm{L}}(\mathrm{St}=795)$, with $\tau_{\eta}$ and $T_{\mathrm{L}}$ the Kolmogorov timescale and the Lagrangian correlation time of the flow, respectively. We computed the rms relative velocity, $\left\langle w^{2}\right\rangle^{1 / 2}$, for all Stokes number pairs $\left(\mathrm{St}_{1}, \mathrm{St}_{2}\right)$ available in the simulation and tested the PP10 model for the general bidisperse case. Here we list our main conclusions.

1. As a special bidisperse case, we examined the relative velocity, $\boldsymbol{w}_{\mathrm{f}}$, between inertial particles and the local flow velocity. We showed that $\boldsymbol{w}_{\mathrm{f}}$ can be roughly estimated as the temporal flow velocity difference, $\Delta \boldsymbol{u}_{\mathrm{T}}(\Delta \tau)$, along the particle trajectory at a time lag, $\Delta \tau$, close to the 
particle friction time $\tau_{\mathrm{p}}$. A simple model is developed for the rms of $\boldsymbol{w}_{\mathrm{f}}$, assuming that the temporal flow velocity correlation on the particle trajectory can be approximated by the Lagrangian correlation function, $\Phi_{\mathrm{L}}$. Adopting a biexponential form for $\Phi_{\mathrm{L}}$, our model is in good agreement with the simulation data. In particular, it predicts that the rms of $\boldsymbol{w}_{\mathrm{f}}$ increases linearly with $\mathrm{St}$ for $\mathrm{St} \ll 1$, scales as $\mathrm{St}^{1 / 2}$ in the inertial range, and finally approaches the flow rms velocity for $\tau_{\mathrm{p}} \gg T_{\mathrm{L}}$. The particle-flow relative velocity is an interesting delimiter that helps confine the relative velocity behavior in the general bidisperse case.

2. We introduced the general formulation of PP10 for the relative velocity of different particles of arbitrary size. The formulation shows that the relative velocity variance is contributed by two terms, named as the generalized acceleration and shear terms because they reduce to the acceleration and shear terms in the Saffman-Turner formula for small particles with $\mathrm{St} \ll 1$. The generalized acceleration term originates from different responses of particles of different sizes to the flow velocities. We established an approximate relation between the generalized acceleration term and the temporal flow velocity difference, $\Delta \boldsymbol{u}_{\mathrm{T}}$, along the trajectory of the larger particle. On the other hand, the generalized shear term represents the contribution from the particles' memory of the spatial flow velocity difference, $\Delta \boldsymbol{u}$, across the distance of the two particles at given times in the past. An analytical expression is derived for the generalized acceleration term, while the generalized shear term is modeled in a way similar to the monodisperse model presented in Paper I, accounting for the combined effects of the particle memory and the separation of particle pairs backward in time. For equal-size particles, the acceleration term vanishes, and only the shear term contributes.

3 . Using our simulation, we computed the rms relative velocity, $\left\langle w^{2}\right\rangle^{1 / 2}$, between particles of varying sizes. We first examined $\left\langle w^{2}\right\rangle^{1 / 2}$ as a function of $\mathrm{St}_{2}$ at fixed values of $\mathrm{St}_{1}$. If $\tau_{\mathrm{p} 1} \lesssim T_{\mathrm{L}}$, the relative velocity shows a dip around $\mathrm{St}_{2} \simeq \mathrm{St}_{1}$, indicating that the velocities of nearby particles of similar size have a tighter correlation than particles of different sizes. The dip disappears for $\tau_{\mathrm{p} 1} \gtrsim T_{\mathrm{L}}$. The generalized shear term dominates the contribution to the rms relative velocity for particles of similar size, while the acceleration term dominates if the Stokes numbers differ by more than a factor of $\simeq 4$.

Defining the ratio, $f \equiv \mathrm{St}_{\ell} / \mathrm{St}_{h}$, between the small $\left(\mathrm{St}_{\ell}\right)$ and large $\left(\mathrm{St}_{h}\right)$ Stokes numbers, we also considered $\left\langle w^{2}\right\rangle^{1 / 2}$ as a function of $\mathrm{St}_{h}$ at fixed values of $0 \leqslant f \leqslant 1$. The limits $f \rightarrow 0$ and $f \rightarrow 1$ correspond to the particle-flow relative velocity and the monodisperse case, respectively. At a fixed $f,\left\langle w^{2}\right\rangle^{1 / 2}$ increases with $\mathrm{St}_{h}$ for $\tau_{\mathrm{p}, \mathrm{h}} \lesssim T_{\mathrm{L}}$, stays roughly constant for $T_{\mathrm{L}} \lesssim \tau_{\mathrm{p}, \mathrm{h}} \lesssim T_{\mathrm{L}} / f$ (or equivalently $f T_{\mathrm{L}} \lesssim \tau_{\mathrm{p}, 1} \lesssim T_{\mathrm{L}}$ ), and finally decreases as $\mathrm{St}_{h}^{-1 / 2}$ for $\tau_{h} \gg T_{\mathrm{L}} / f$. For any value of $f, \mathrm{a} \mathrm{St}_{h}^{1 / 2}$ scaling is predicted if the larger friction time, $\tau_{\mathrm{p}, \mathrm{h}}$, is within the inertial range of the flow. This $\mathrm{St}_{h}^{1 / 2}$ scaling will have to be verified in future simulations with higher resolutions. At a given $\mathrm{St}_{h}$, $\left\langle w^{2}\right\rangle^{1 / 2}$ increases with decreasing $f$ due to the increase of the acceleration contribution, which starts to dominate at $f \lesssim 1 / 4$.

The generalized acceleration contribution is independent of the distance, $r$, and thus reduces the $r$ dependence of the relative velocity between small, different particles, making it easier to achieve numerical convergence for the collision statistics of point-like particles at $r \rightarrow 0$.

The prediction of the PP10 model is in good agreement with the simulation data. The largest discrepancy occurs for $f=(1 / 2)$ and $\mathrm{St}_{h}$ in the inertial range, where the model underestimates the rms relative velocity by $15 \%-20 \%$. At other values of $f$, the discrepancy between our model and the simulation is $<10 \%$. This confirms the validity of the physical picture revealed by our model.

We emphasize that the theoretical modeling of the rms relative velocity is important for understanding the fundamental physics, even though its practical use is limited. In future work, we will focus on establishing statistical measures or tools that can be applied to model dust particle collisions in protoplanetary turbulence. We have started an effort in an ongoing paper (L. Pan \& P. Padoan 2014, in preparation) to explore the collision kernel in the general bidisperse case, accounting for turbulence-induced collision velocity and the effect of turbulent clustering. In the next paper of this series, we will systematically examine the probability distribution of the collision velocity, which is needed to determine the fractions of collisions leading to sticking, bouncing, or fragmentation. Due to the limited resolution, the simulated flow in the current work has only a short inertial range, and our model prediction for particles in the inertial range remains to be tested and validated. Future simulations at higher resolutions are being planned to obtain accurate measurements for the collision statistics of inertialrange particles.

Resources supporting this work were provided by the NASA High-End Computing (HEC) Program through the NASA Advanced Supercomputing (NAS) Division at Ames Research Center and by the Port d'Informació Científica (PIC), Spain, maintained by a collaboration of the Institut de Fsica d'Altes Energies (IFAE) and the Centro de Investigaciones Energticas, Medioambientales y Tecnolgicas (CIEMAT). L.P. is supported by a Clay Fellowship at Harvard-Smithsonian Center for Astrophysics. P.P. acknowledges support by the FP7-PEOPLE-2010-RG grant PIRG07-GA-2010-261359.

\section{APPENDIX A}

\section{THE FORMULATION OF PAN \& PADOAN (2010)}

We review the general PP10 formulation for particles of different sizes. It follows from Equation (9) that the particle velocity structure tensor, $S_{\mathrm{p} i j}$, has four terms, $\left\langle v_{i}^{(1)} v_{j}^{(1)}\right\rangle,-\left\langle v_{i}^{(1)} v_{j}^{(2)}\right\rangle$, $-\left\langle v_{i}^{(2)} v_{j}^{(1)}\right\rangle$, and $\left\langle v_{i}^{(2)} v_{j}^{(2)}\right\rangle$. Inserting the formal solution, Equation (2), for $\boldsymbol{v}^{(1)}$ and/or $\boldsymbol{v}^{(2)}$ into each term yields a double integral. The terms $\left\langle v_{i}^{(1)} v_{j}^{(1)}\right\rangle$ and $\left\langle v_{i}^{(2)} v_{j}^{(2)}\right\rangle$ correspond to the one-particle velocity variances, which have been evaluated in Section 2 of Paper I. For particle $1,\left\langle v_{i}^{(1)} v_{j}^{(1)}\right\rangle$ at $t=0$ can be calculated as

$$
\begin{aligned}
\left\langle v_{i}^{(1)} v_{j}^{(1)}\right\rangle= & \int_{-\infty}^{0} \frac{d \tau}{\tau_{\mathrm{p} 1}} \int_{-\infty}^{0} \frac{d \tau^{\prime}}{\tau_{\mathrm{p} 1}} B_{i j}^{(1)}\left(\tau, \tau^{\prime}\right) \\
& \times \exp \left(\frac{\tau}{\tau_{\mathrm{p} 1}}\right) \exp \left(\frac{\tau^{\prime}}{\tau_{\mathrm{p} 1}}\right),
\end{aligned}
$$

where $B_{i j}^{(1)}=\left(\equiv\left\langle u_{i}^{(1)}(\tau) u_{j}^{(1)}\left(\tau^{\prime}\right)\right\rangle\right)$ is the trajectory correlation tensor of particle 1 (see Section 2). A similar equation can be derived for $\left\langle v_{i}^{(2)} v_{j}^{(2)}\right\rangle$, which depends on $B_{i j}^{(2)}$. 
The cross-terms, $\left\langle v_{i}^{(1)} v_{j}^{(2)}\right\rangle$ and $\left\langle v_{i}^{(2)} v_{j}^{(1)}\right\rangle$, involve the memories of the flow velocities by both particles. For the first cross-term, we have

$$
\begin{aligned}
\left\langle v_{i}^{(1)} v_{j}^{(2)}\right\rangle= & \int_{-\infty}^{0} \frac{d \tau}{\tau_{\mathrm{p} 1}} \int_{-\infty}^{0} \frac{d \tau^{\prime}}{\tau_{\mathrm{p} 2}}\left\langle u_{i}^{(1)}(\tau) u_{j}^{(2)}\left(\tau^{\prime}\right)\right\rangle \\
& \times \exp \left(\frac{\tau}{\tau_{\mathrm{p} 1}}\right) \exp \left(\frac{\tau^{\prime}}{\tau_{\mathrm{p} 2}}\right)
\end{aligned}
$$

where $\left\langle u_{i}^{(1)}(\tau) u_{j}^{(2)}\left(\tau^{\prime}\right)\right\rangle$ is the correlation of the flow velocities seen by particles 1 and 2 at $\tau$ and $\tau^{\prime}$, respectively. A similar integral equation exists for $\left\langle v_{i}^{(2)} v_{j}^{(1)}\right\rangle$, which contains $\left\langle u_{j}^{(1)}(\tau) u_{i}^{(2)}\left(\tau^{\prime}\right)\right\rangle$. The sum of $\left\langle u_{i}^{(1)}(\tau) u_{j}^{(2)}\left(\tau^{\prime}\right)\right\rangle$ and $\left\langle u_{j}^{(1)}(\tau) u_{i}^{(2)}\left(\tau^{\prime}\right)\right\rangle$ can be written as

$$
\begin{aligned}
\left\langle u_{i}^{(1)}(\tau) u_{j}^{(2)}\left(\tau^{\prime}\right)\right\rangle & +\left\langle u_{j}^{(1)}(\tau) u_{i}^{(2)}\left(\tau^{\prime}\right)\right\rangle=-S_{\mathrm{T} i j}\left(\boldsymbol{r}, \tau, \tau^{\prime}\right)+B_{i j}^{(1)}\left(\tau, \tau^{\prime}\right) \\
& +B_{i j}^{(2)}\left(\tau, \tau^{\prime}\right)-C_{i j}\left(\boldsymbol{r}, \tau, \tau^{\prime}\right),
\end{aligned}
$$

where $S_{\mathrm{T} i j}\left(\boldsymbol{r}, \tau, \tau^{\prime}\right)$ is named as the trajectory structure tensor. It is defined as

$$
S_{\mathrm{T} i j}\left(\boldsymbol{r}, \tau, \tau^{\prime}\right)=\left\langle\left[u_{i}^{(1)}(\tau)-u_{i}^{(2)}(\tau)\right]\left[u_{j}^{(1)}\left(\tau^{\prime}\right)-u_{j}^{(2)}\left(\tau^{\prime}\right)\right]\right\rangle,
$$

which represents the correlation of the flow velocity differences seen by the two particles at two times. It depends on the particle separation $\boldsymbol{r}$ at $t=0$ through the constraint $\boldsymbol{X}^{(2)}(0)-$ $\boldsymbol{X}^{(1)}(0)=\boldsymbol{r}$.

The last term in Equation (A3) is defined as $C_{i j}\left(\boldsymbol{r}, \tau, \tau^{\prime}\right) \equiv$ $\left\langle u_{j}^{(1)}\left(\tau^{\prime}\right) u_{i}^{(2)}(\tau)\right\rangle-\left\langle u_{j}^{(1)}(\tau) u_{i}^{(2)}\left(\tau^{\prime}\right)\right\rangle$. Since $C_{i j}$ is antisymmetric under the exchange of $\tau$ and $\tau^{\prime}$, it is easy to see that $\int d \tau / \tau_{\mathrm{p} 1} \int d \tau^{\prime} / \tau_{\mathrm{p} 2} C_{i j} \exp \left(\tau / \tau_{\mathrm{p} 1}\right) \exp \left(\tau^{\prime} / \tau_{\mathrm{p} 2}\right)$ is zero for equalsize particles with $\tau_{\mathrm{p} 1}=\tau_{\mathrm{p} 2}$. It can also be shown that $C_{i j}=0$ if both particles are small with $\mathrm{St}_{1,2} \ll 1$. On the other hand, the term is not expected to exactly vanish for particles of arbitrarily different sizes. For example, given the particle distance at $\tau,\left\langle u_{j}^{(1)}\left(\tau^{\prime}\right) u_{i}^{(2)}(\tau)\right\rangle$ depends on the trajectory of particle 1 , or more precisely, the flow velocity decorrelation along its trajectory from $\tau$ to $\tau^{\prime}$, while $\left\langle u_{j}^{(1)}(\tau) u_{i}^{(2)}\left(\tau^{\prime}\right)\right\rangle$ is controlled by the trajectory of particle 2 . Therefore, a difference may exist between $\left\langle u_{j}^{(1)}\left(\tau^{\prime}\right) u_{i}^{(2)}(\tau)\right\rangle$ and $\left\langle u_{j}^{(1)}(\tau) u_{i}^{(2)}\left(\tau^{\prime}\right)\right\rangle$ due to the different temporal statistics along the trajectories of the two particles. However, Paper I showed that the Lagrangian and Eulerian temporal correlation functions in our simulated flow are close to each other, meaning that the decorrelation of the flow velocity along a trajectory of a small particle in the $\mathrm{St} \ll 1$ limit may be similar to that for a large particle with $\tau_{\mathrm{p}} \gg T_{\mathrm{L}}$. Based on this extreme case, one could assume that, qualitatively, $\left\langle u_{j}^{(1)}\left(\tau^{\prime}\right) u_{i}^{(2)}(\tau)\right\rangle \simeq\left\langle u_{j}^{(1)}(\tau) u_{i}^{(2)}\left(\tau^{\prime}\right)\right\rangle$ for particles of any different sizes. We thus neglect $C_{i j}$ in our model, even though the quantitative accuracy of the assumption is unclear. The $C_{i j}$ term was ignored in PP10, where it was found that, without $C_{i j}$, the model prediction is in good agreement with the simulation results of Zhou et al. (2001) for the bidisperse case.

Adding the four terms of $S_{\mathrm{p} i j}$ together, rearranging the integrals using Equation (A3), and neglecting $C_{i j}$, the particle structure tensor can be written as two terms,

$$
S_{\mathrm{p} i j}(\boldsymbol{r})=\mathcal{A}_{i j}+\mathcal{S}_{i j}
$$

where $\mathcal{A}_{i j}$ and $\mathcal{S}_{i j}$, named the generalized acceleration and shear terms, respectively (PP10), reduce to the acceleration and shear terms in the S-T limit, Equation (12), for $\mathrm{St}_{1,2} \ll 1$, respectively (see Sections 3.1 and 3.2.1).

The generalized acceleration term is given by

$$
\begin{aligned}
\mathcal{A}_{i j}= & \int_{-\infty}^{0} \frac{d \tau}{\tau_{\mathrm{p} 1}} \int_{-\infty}^{0} \frac{d \tau^{\prime}}{\tau_{\mathrm{p} 1}} B_{i j}^{(1)}\left(\tau, \tau^{\prime}\right) \exp \left(\frac{\tau}{\tau_{\mathrm{p} 1}}\right) \exp \left(\frac{\tau^{\prime}}{\tau_{\mathrm{p} 1}}\right) \\
& -\int_{-\infty}^{0} \frac{d \tau}{\tau_{\mathrm{p} 1}} \int_{-\infty}^{0} \frac{d \tau^{\prime}}{\tau_{\mathrm{p} 2}}\left[B_{i j}^{(1)}\left(\tau, \tau^{\prime}\right)+B_{i j}^{(2)}\left(\tau, \tau^{\prime}\right)\right] \\
& \times \exp \left(\frac{\tau}{\tau_{\mathrm{p} 1}}\right) \exp \left(\frac{\tau^{\prime}}{\tau_{\mathrm{p} 2}}\right)+\int_{-\infty}^{0} \frac{d \tau}{\tau_{\mathrm{p} 2}} \int_{-\infty}^{0} \frac{d \tau^{\prime}}{\tau_{\mathrm{p} 2}} \\
& \times B_{i j}^{(2)}\left(\tau, \tau^{\prime}\right) \exp \left(\frac{\tau}{\tau_{\mathrm{p} 2}}\right) \exp \left(\frac{\tau^{\prime}}{\tau_{\mathrm{p} 2}}\right)
\end{aligned}
$$

Clearly, $\mathcal{A}_{i j}$ vanishes if $\tau_{\mathrm{p} 1}=\tau_{\mathrm{p} 2} . \mathcal{A}_{i j}$ is independent of $\boldsymbol{r}$, as it depends only on the flow velocity statistics $\left(B_{i j}^{(1)}\right.$ and $\left.B_{i j}^{(2)}\right)$ along individual trajectories of the two particles.

The generalized shear term reads

$$
\mathcal{S}_{i j}=\int_{-\infty}^{0} \frac{d \tau}{\tau_{\mathrm{p} 1}} \int_{-\infty}^{0} \frac{d \tau^{\prime}}{\tau_{\mathrm{p} 2}} S_{\mathrm{T} i j}\left(\boldsymbol{r} ; \tau, \tau^{\prime}\right) \exp \left(\frac{\tau}{\tau_{\mathrm{p} 1}}\right) \exp \left(\frac{\tau^{\prime}}{\tau_{\mathrm{p} 2}}\right),
$$

which represents the contribution from the particles' memory of the flow velocity difference they saw in the past. In the limit $\tau_{\mathrm{p} 1}, \tau_{\mathrm{p} 2} \rightarrow 0$, the exponential cutoffs can be viewed as delta functions, and we thus have $\mathcal{S}_{i j} \rightarrow S_{\mathrm{T} i j}(\boldsymbol{r} ; 0,0)=S_{i j}(\boldsymbol{r})$ with $S_{i j}(\boldsymbol{r})$ the flow structure tensor (PP10 and Paper I). Equation (A7) thus reproduces the shear terms in the S-T limit (Equation (12)).

\section{APPENDIX B}

\section{MODELING THE GENERALIZED SHEAR TERM}

We list the assumptions made to calculate Equation (23) for the generalized shear term, $\mathcal{S}_{i j}$. In an isotropic flow, the Eulerian structure tensor $S_{i j}(\boldsymbol{R})$ in Equation (23) is written as $S_{\mathrm{nn}}(R) \delta_{i j}+$ $\left[S_{\mathrm{ll}}(R)-S_{\mathrm{nn}}(R)\right]\left(R_{i} R_{j} / R^{2}\right)$, with $S_{\mathrm{ll}}$ and $S_{\mathrm{nn}}$ the longitudinal and transverse structure functions. To evaluate the angular average of $S_{i j}(\boldsymbol{R})$, we assume that the direction of $\boldsymbol{R}$ is isotropic and random, resulting in $\left\langle S_{i j}(\boldsymbol{R})\right\rangle_{\text {ang }}=(1 / 3)\left[S_{11}(R)+2 S_{\mathrm{nn}}(R)\right] \delta_{i j}$ (which is Equation (24) in the text). This assumption suggests the shear contributions for the radial and tangential rms relative speeds are equal for all particles. Under this assumption, the shear terms in the S-T limit $\left(\mathrm{St}_{1,2} \ll 1\right)$ are given by $\bar{\epsilon} / 9 v r^{2}$ for both $\left\langle w_{\mathrm{r}}^{2}\right\rangle$ and $\left\langle w_{\mathrm{t}}^{2}\right\rangle$. In PP10 and Paper I, another assumption was adopted for the angular average (see Equation (26) in Paper I), which exactly reproduces the shear terms for the radial and tangential variances in the S-T formula (Equation (12)) for $\mathrm{St}_{1,2} \ll 1$. This second assumption is, however, in poorer agreement with our simulation data for particles with $\mathrm{St} \gtrsim 0.1$. We mainly consider Equation (24) for $\left\langle S_{i j}(\boldsymbol{R})\right\rangle_{\text {ang }}$ in this paper.

In Paper I we considered both a single- and a bi-exponential form for the temporal correlation function $\Phi_{2}$ in Equation (23), i.e.,

$$
\Phi_{2}(\Delta \tau, R)=\exp \left(-\frac{-|\Delta \tau|}{T(R)}\right)
$$


and

$$
\begin{aligned}
\Phi_{2}(\Delta \tau, R)= & \frac{1}{2 \sqrt{1-2 z^{2}}}\left\{\left(1+\sqrt{1-2 z^{2}}\right) \exp \left[-\frac{2|\Delta \tau|}{\left(1+\sqrt{1-2 z^{2}}\right) T(R)}\right]\right. \\
& \left.-\left(1-\sqrt{1-2 z^{2}}\right) \exp \left[-\frac{2|\Delta \tau|}{\left(1-\sqrt{1-2 z^{2}}\right) T(R)}\right]\right\}, \quad(\mathrm{B} 2)
\end{aligned}
$$

where the correlation time $T(R)$ corresponds to the eddy turnover time at the scale $R$.

The typical particle distance, $R$, between $\tau$ and $\tau^{\prime}$ was approximated by

$$
R\left(\tau, \tau^{\prime}\right)=\left[d(\tau) d\left(\tau^{\prime}\right)\right]^{1 / 2},
$$

where $d(\tau)$ and $d\left(\tau^{\prime}\right)$ are the rms particle distances at $\tau$ and $\tau^{\prime}$. It was motivated by the fact that $S_{\mathrm{T} i j}$ is zero if either $d(\tau)$ or $d\left(\tau^{\prime}\right)$ is zero (PP10). The backward separation of inertial particle pairs has not been explored until recently (Bragg et al. 2014). The simulation results of Bec et al. (2010) for the forward-in-time dispersion of equal-size particles were used as a guide for the assumption for the backward separation (PP10). We adopted a two-phase behavior. In the first phase, the particle pairs separate ballistically,

$$
d^{2}(\tau)=r^{2}+\left\langle w^{2}\right\rangle \tau^{2}
$$

where $\left\langle w^{2}\right\rangle$ is the $3 \mathrm{D}$ relative velocity variance. The second phase follows Richardson's law,

$$
d^{2}(\tau) \simeq g \bar{\epsilon}|\tau|^{3},
$$

where $g$ is the Richardson constant (Paper I). For equal-size particles, the transition between the two phases was assumed to occur at a friction time or so, $\tau \simeq-\tau_{\mathrm{p}}$, based on the results of Bec et al. (2010). We thus connect the behaviors at a transition time, $\tau_{\mathrm{c}}=-\tau_{\mathrm{p}}$. A number of uncertainties in the assumed two-phase behavior were pointed out and justified in Paper I for equal-size particles. In a recent study, Bragg et al. (2014) examined both forward and backward-in-time separation behaviors of inertial particle pairs and very briefly mentioned a possible problem in our assumption based on the forward separation behavior. Apparently, their claim of the potential accuracy problem in our model prediction due to the adopted separation behavior is discussed in a forthcoming paper that has not yet been published. We will consider/address their comments in future works. We will adopt the same two-phase behavior for the bidisperse case. An additional uncertainty here is that it is not clear how long the ballistic phase lasts, as the two friction times are different. Following PP10, we assume that the transition occurs at $\tau_{\mathrm{c}}=-\left(\tau_{\mathrm{p} 1}+\tau_{\mathrm{p} 2}\right) / 2$. This may be questionable for particles of very different sizes. It, however, does not present a severe problem because, in that case, it is the generalized acceleration term that gives the dominant contribution to the relative velocity (see Section 6.1). For the Richardson constant, $g$, we will adopt the values used in Paper I that best fit the data for identical particles.

To calculate the generalized shear term, we also need the flow structure functions, $S_{11}$ and $S_{\mathrm{nn}}$, as functions of the length scale, $\ell$ (see Equation (27) in Section 3.3). Following Paper I, we adopt the following connecting formulas,

$$
\begin{aligned}
S_{11}(\ell)= & 2 u^{\prime 2}\left[1-\exp \left(-\frac{(\ell / \eta)}{\left(15 C_{\mathrm{K}}\right)^{3 / 4}}\right)\right]^{4 / 3} \\
& \times\left[\frac{(\ell / \eta)^{4}}{(\ell / \eta)^{4}+\left(2 u^{\prime 2} / C_{\mathrm{K}} u_{\eta}^{2}\right)^{6}}\right]^{1 / 6},
\end{aligned}
$$

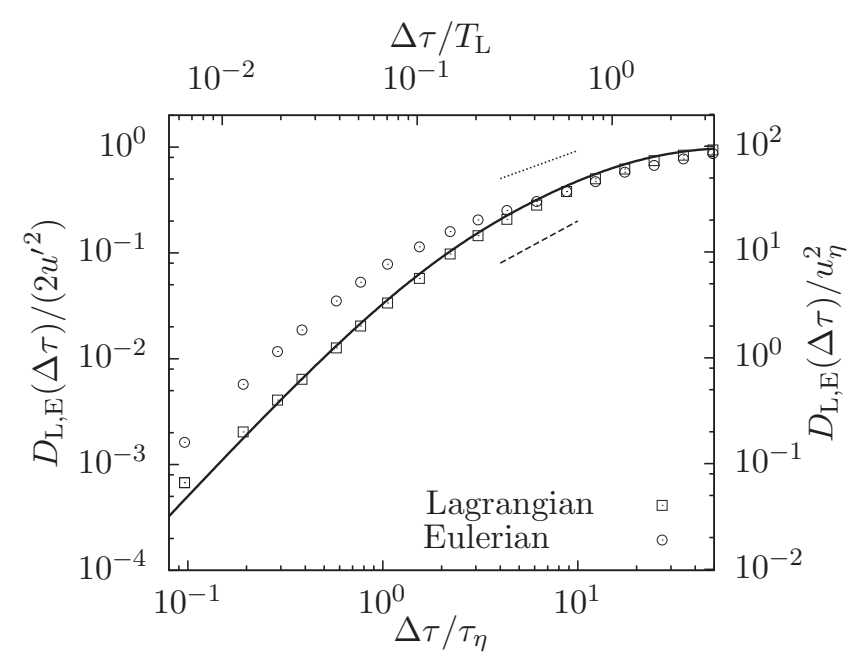

Figure 11. Lagrangian (squares) and Eulerian (circles) temporal structure functions. The left and right $y$ axes normalize these functions to $2 u^{\prime 2}$ and $u_{\eta}^{2}$, respectively. The solid line corresponds to $1-\Phi_{\mathrm{L}}(\Delta \tau)$ using a bi-exponential $\Phi_{\mathrm{L}}$ (Equation (7)) with $z=0.3$ and $T_{\mathrm{L}}=15 \tau_{\eta}$. The dashed and dotted line segments denote a linear and a $(\Delta \tau)^{2 / 3}$ scaling, respectively, for $\Delta \tau$ in the inertial range.

$$
\begin{aligned}
S_{\mathrm{nn}}(\ell)= & 2 u^{\prime 2}\left[1-\exp \left(-\frac{(\ell / \eta)^{4 / 3}}{\left(15 C_{\mathrm{Kn}} / 2\right)}\right)\right] \\
& \times\left[\frac{(\ell / \eta)^{4}}{(\ell / \eta)^{4}+\left(2 u^{\prime 2} / C_{\mathrm{Kn}} u_{\eta}^{2}\right)^{6}}\right]^{1 / 6} .
\end{aligned}
$$

With $C_{\mathrm{K}}=2$ and $C_{\mathrm{Kn}}=2.5$, the two formulas fit the measured structure functions in our simulated flow well. The correlation timescale, $T$, in Equations (B1) and (B2) for $\Phi_{2}$ is also obtained by a connecting formula (Zaichik et al. 2006),

$$
\begin{aligned}
T(\ell)=T_{\mathrm{L}} & {\left[1-\exp \left(-\left(\frac{C_{\mathrm{T}}}{\sqrt{5}}\right)^{3 / 2}(\ell / \eta)\right)\right]^{-2 / 3} } \\
& \times\left[\frac{(\ell / \eta)^{4}}{(\ell / \eta)^{4}+\left(T_{\mathrm{L}} /\left(C_{\mathrm{T}} \tau_{\eta}\right)\right)^{6}}\right]^{1 / 6}
\end{aligned}
$$

with the parameter $C_{\mathrm{T}}$ set to 0.4 (Paper I).

\section{APPENDIX C \\ LAGRANGIAN AND EULERIAN TEMPORAL STRUCTURE FUNCTIONS}

As discussed in Sections 2 and 3, the particle-flow relative velocity and the generalized acceleration term in the bidisperse case depend on the temporal flow velocity difference, $\Delta_{\mathrm{T}} \boldsymbol{u}(\Delta \tau)$, along particle trajectories. As small particles more or less follow Lagrangian tracers, it is helpful to examine the flow velocity difference $\Delta \boldsymbol{u}_{\mathrm{L}}(\Delta \tau) \equiv \boldsymbol{u}\left(\boldsymbol{X}_{\mathrm{L}}(t+\Delta \tau), t+\Delta \tau\right)-\boldsymbol{u}\left(\boldsymbol{X}_{\mathrm{L}}(t), t\right)$ as a function of the time lag, $\Delta \tau$, along Lagrangian trajectories $\boldsymbol{X}_{\mathrm{L}}(t)$. We also consider the Eulerian temporal velocity difference $\Delta \boldsymbol{u}_{\mathrm{E}}(\Delta \tau) \equiv \boldsymbol{u}(\boldsymbol{x}, t+\Delta \tau)-\boldsymbol{u}(\boldsymbol{x}, t)$ at fixed points, $\boldsymbol{x}$, as the flow velocity seen by large particles with $\tau_{\mathrm{p}} \gtrsim T_{\mathrm{L}}$ may be better described as Eulerian (Paper I).

We define Lagrangian/Eulerian structure tensors as $D_{\mathrm{L}, \mathrm{E} i j}(\Delta \tau) \equiv\left\langle\Delta \boldsymbol{u}_{\mathrm{L}, \mathrm{E} i}(\Delta \tau) \Delta \boldsymbol{u}_{\mathrm{L}, \mathrm{E} j}(\Delta \tau)\right\rangle$, and by isotropy $D_{\mathrm{L}, \mathrm{E} i j}(\Delta \tau)=D_{\mathrm{L}, \mathrm{E}}(\Delta \tau) \delta_{i j}$. By definition, $D_{\mathrm{L}, \mathrm{E}}=2 u^{\prime 2}(1-$ $\left.\Phi_{\mathrm{L}, \mathrm{E}}\right)$, where $\Phi_{\mathrm{L}, \mathrm{E}}$ are the temporal correlation functions of Lagrangian and Eulerian velocities (see Paper I). Figure 11 shows 

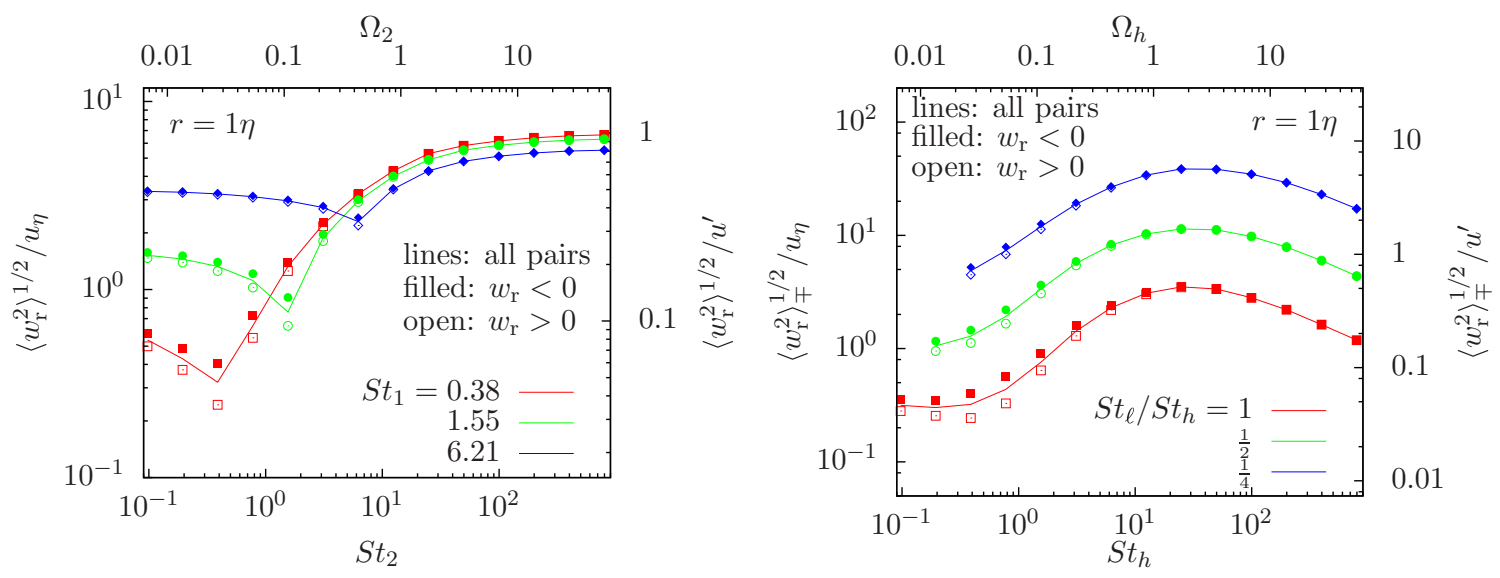

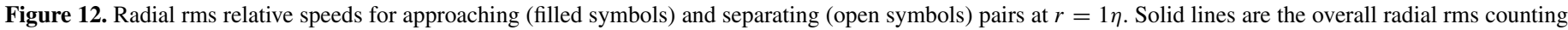

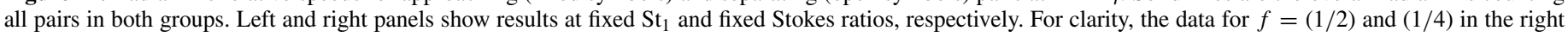
panel are shifted upward by a factor of three and nine, respectively.

(A color version of this figure is available in the online journal.)

$D_{\mathrm{L}}$ (squares) and $D_{\mathrm{E}}$ (circles) as a function of $\Delta \tau$. The figure is equivalent to Figure 2 in Paper I for $\Phi_{\mathrm{L}, \mathrm{E}}$. The solid line plots $\left(1-\Phi_{\mathrm{L}}\right)$ using a bi-exponential $\Phi_{\mathrm{L}}$ (Equation (7)) with $z=0.3$ and $T_{\mathrm{L}}=15 \tau_{\eta}$, consistent with the values obtained in Figure 2 of Paper I. Except at the smallest $\Delta \tau$, the line provides a good fit to the data. The measured values of $z$ and $T_{\mathrm{L}}$ indicate a Taylor microtimescale of $4.3 \tau_{\eta}$ and a $1 \mathrm{D} \mathrm{rms}$ acceleration, $a$, of $2.2 \tau_{\eta}^{-1}$.

The dashed and dotted line segments denote a linear and a $(\Delta \tau)^{2 / 3}$ scaling. In the inertial range, $D_{\mathrm{L}}(\Delta \tau)$ is expected to be $\simeq \bar{\epsilon} \Delta \tau$ from the Lagrangian version of Kolmogorov's similarity theory (Monin \& Yaglom 1975), while the $(\Delta \tau)^{2 / 3}$ scaling for $D_{\mathrm{E}}(\Delta \tau)$ follows from the random Taylor hypothesis that connects the Eulerian temporal and spatial structure functions (Tennekes 1975). The inertial range of our flow is short especially for the Lagrangian structure function. A linear scaling is barely seen in $D_{\mathrm{L}}$. At $\Delta \tau$ below the Taylor microtimescale $\tau_{\mathrm{T}} \simeq 4.3 \tau_{\eta}, D_{\mathrm{L}}$ is expected to be $a^{2}(\Delta \tau)^{2}$, but we see the slope of the square data points at the smallest $\Delta \tau\left(\simeq 0.1 \tau_{\eta}\right)$ in the figure is slightly shallower than $(\Delta \tau)^{2}$. An exact $(\Delta \tau)^{2}$ scaling may appear when one extends the measurement to $\Delta \tau$ below $0.1 \tau_{\eta}$. We note that $D_{\mathrm{L}}$ merges with $D_{\mathrm{E}}$ at $\Delta \tau \simeq 8 \tau_{\eta}$, suggesting that we can use $D_{\mathrm{L}}$ in our model for particles with $\tau_{\mathrm{p}} \gtrsim T_{\mathrm{L}} \simeq 15 \tau_{\eta}$, even though trajectories of these large particles would significantly deviate from tracer particles. In other words, this provides a justification for approximating $\Delta_{\mathrm{T}} \boldsymbol{u}$ by $\Delta_{\mathrm{L}} \boldsymbol{u}$ for all particles.

\section{APPENDIX D}

\section{APPROACHING AND SEPARATING PARTICLE PAIRS}

As in Paper I, we split particle pairs at given distances into two groups with negative $\left(w_{\mathrm{r}}<0\right)$ and positive $\left(w_{\mathrm{r}}>0\right)$ radial relative speed, corresponding to particle pairs approaching and separating from each other, respectively. We named them as minus and plus groups. Although only the minus group is relevant for collisions, it is of theoretical interest to compare the two groups. Figure 12 plots the radial rms relative speeds for particle pairs in the minus $\left(\left\langle w_{\mathrm{r}}^{2}\right\rangle_{-}^{1 / 2}\right.$; filled symbols $)$ and plus $\left(\left\langle w_{\mathrm{r}}^{2}\right\rangle_{+}^{1 / 2}\right.$; open symbols) groups at a distance of $r=1 \eta$.

In Paper I, we showed that, for identical particles with $\mathrm{St} \lesssim 6.2$, the rms relative speed in the minus group is larger than in the plus group. For St $\ll 1$ particles, one expects the relative speed to inherit such an asymmetry from the flow (see
Appendix B of Paper I). As St increases, the asymmetry is first amplified and then decreases at St $\gtrsim 0.4$ (see the $f=1$ data in the right panel). The amplification is due to the fact that approaching pairs come from a larger distance in the near past than separating ones. This tends to make the relative speed in the minus group larger because the relative velocity of identical particles is determined by their memory of the flow velocity difference in the past, which is larger at larger particle separation. For larger particles with $\mathrm{St} \gtrsim 0.4$, separating pairs would move past each other within a friction time in the past, and their separation then starts to increase backward in time. Consequently, the difference in the particle distance at a friction time in the past for minus and plus pairs decreases, causing the asymmetry to decrease. It finally disappears for $\mathrm{St} \gtrsim 6.2$, as the amplitude of the particle separation at a friction time ago becomes insensitive to $r$ or the condition in the near past (Paper I).

The left panel of Figure 12 shows that, for each $\mathrm{St}_{1}$, the difference between the two groups is largest for identical particles and decreases as $\mathrm{St}_{2}$ moves away from the dip center. This is because the contribution of the generalized acceleration term does not depend on whether the particles are approaching or separating. The acceleration term is determined by the flow velocity statistics along the individual trajectories of the two particles (see Section 3.2.1 and Figure 1) and thus independent of their relative motions. Therefore, the asymmetry of the plus and minus groups decreases when the acceleration contribution increases. This is also seen in the right panel where the asymmetry is weaker at smaller $f$. If one Stokes number is larger than 6.21, the asymmetry disappears for any bidisperse case. As mentioned in Paper I, the asymmetry in $\left\langle w_{\mathrm{r}}^{2}\right\rangle_{-}^{1 / 2}$ and $\left\langle w_{\mathrm{r}}^{2}\right\rangle_{+}^{1 / 2}$ is related to the spatial clustering of the particles. A smaller difference in $\left\langle w_{\mathrm{r}}^{2}\right\rangle_{-}^{1 / 2}$ and $\left\langle w_{\mathrm{r}}^{2}\right\rangle_{+}^{1 / 2}$ for particles of different sizes suggests a weaker clustering in the bidisperse case (Pan et al. 2011).

The asymmetry tends to decrease with decreasing $r$, and the decrease is faster for the bidisperse case than for the monodisperse case. The asymmetry would disappear at sufficiently small $r$ once the acceleration term dominates. We also examined the tangential $\left(\left\langle w_{\mathrm{t}}^{2}\right\rangle_{\mp}^{1 / 2}\right)$ and the 3D $\left(\left\langle w^{2}\right\rangle_{\mp}^{1 / 2}\right)$ rms relative speeds in the minus and plus groups and found a similar asymmetry to the case of the radial component. Paper I showed that the radial and tangential rms relative speeds of approaching pairs are about equal (i.e., $\left\langle w_{\mathrm{r}}^{2}\right\rangle_{-}^{1 / 2} \simeq\left\langle w_{\mathrm{t}}^{2}\right\rangle_{-}^{1 / 2}$ ) for all particles in our 
simulation. The same is true for the bidisperse case, as the acceleration term has the effect of equalizing the radial and tangential components.

\section{REFERENCES}

Abrahamson, J. 1975, ChEnS, 30, 1371

Ayala, O., Grabowski, W. W., \& Wang, L.-P. 2007, JCoPh, 225, 51

Bec, J., Biferale, L., Lanotte, A. S., Scagliarini, A., \& Toschi, F. 2010, JFM, 645,497

Birnstiel, T., Ormel, C. W., \& Dullemond, C. P. 2011, A\&A, 525, 11

Blum, J., \& Wurm, G. 2008, ARA\&A, 46, 21

Bragg, A. D., Ireland, P. J., \& Collins, L. R. 2014, arXiv:1403.5502v1

Carballido, A. 2011, Icar, 211, 876

Cuzzi, J. N., \& Hogan, R. C. 2003, Icar, 164, 127

Derivich, I. V. 2006, IJHMT, 49, 4290

Dullemond, C. P., \& Dominik, C. 2005, A\&A, 434, 971

Falkovich, G., Fouxon, A., \& Stepanov, M. G. 2002, Natur, 419, 151

Garaud, P., Meru, F., Galvagni, M., \& Olczak, C. 2013, ApJ, 764, 146

Guttler, C., Blum, J., Zsom, A., Ormel, C. W., \& Dullemond, C. P. 2010, A\&A, 513, A56

Hubbard, A. 2012, MNRAS, 426, 784

Hubbard, A. 2013, MNRAS, 432, 1274

Johansen, A., \& Youdin, A. N. 2007, ApJ, 662, 627

Kruis, F. E., \& Kusters, K. A. 1997, ChEnC, 158, 201

Markiewicz, W. J., Mizuno, H., \& Voelk, H. J. 1991, A\&A, 242, 286

Monin, A. S., \& Yaglom, A. M. 1975, Statistical Fluid Mechanics: Mechanics of Turbulence, Vol. 2 (Cambridge, MA: MIT Press)
Morfill, G. E., Durisen, R. H., \& Turner, G. W. 1998, Icar, 134, 180

Ormel, C. W., \& Cuzzi, J. N. 2007, A\&A, 466, 413

Ormel, C. W., Cuzzi, J. N., \& Tielens, A. G. G. M. 2008, ApJ, 679, 1588

Pan, L., \& Padoan, P. 2010, JFM, 661, 73 (PP10)

Pan, L., \& Padoan, P. 2013, ApJ, 776, 12 (Paper I)

Pan, L., Padoan, P., Scalo, J., Kritsuk, A. G., \& Norman, M. L. 2011, ApJ, 740,21

Pan, L., \& Scannapieco, E. 2011, PhRvE, 2011, 83, 045302

Paque, J. M., \& Cuzzi, J. N. 1997, LPI, 28, 1071

Saffman, P. G., \& Turner, J. S. 1956, JFM, 1, 16

Tennekes, H. 1975, JFM, 67, 561

Völk, H. J., Jones, F. C., Morfill, G. E., \& Roeser, S. 1980, A\&A, 85, 316

Wang, L.-P., Wexler, A. S., \& Zhou, Y. 2000, JFM, 415, 117

Weidenschilling, S. J. 1984, Icar, 60, 553

Wilkinson, M., Mehlig, B., \& Bezuglyy, V. 2006, PhRvL, 97, 048501

Windmark, F., Birnstiel, T., Ormel, C. W., \& Dullemond, C. P. 2012, A\&A, 544, L16

Yuu, S. 1984, AIChE, 30, 802

Zaichik, L. I., \& Alipchenkov, V. M. 2003, PhFl, 15, 1776

Zaichik, L. I., \& Alipchenkov, V. M. 2009, NJPh, 11, 103018

Zaichik, L. I., Alipchenkov, V. M., \& Sinaiski, E. G. 2008, Particles in Turbulent Flows (Weinheim: Wiley-VCH)

Zaichik, L. I., Simonin, O., \& Alipchenkov, V. M. 2003, PhFl, 15, 2995

Zaichik, L. I., Simonin, O., \& Alipchenkov, V. M. 2006, PhFl, 18, 035110

Zhou, Y., Wexler, A. S., \& Wang, L.-P. 2001, JFM, 433, 77

Zsom, A., Ormel, C. W., Dullemond, C. P., \& Henning, T. 2011, A\&A, 534, A73

Zsom, A., Ormel, C. W., Güttler, C., Blum, J., \& Dullemond, C. P. 2010, A\&A, 513, A57 